\title{
DAILY SNOW DEPTH MEASUREMENTS FROM 195 STATIONS IN THE UNITED STATES
}

\author{
D. R. Easterling - P. Jamason - D. P. Bowman - P. Y. Hughes - E. H. Mason
}

National Climatic Data Center

Linda J. Allison, editor

Carbon Dioxide Information Analysis Center
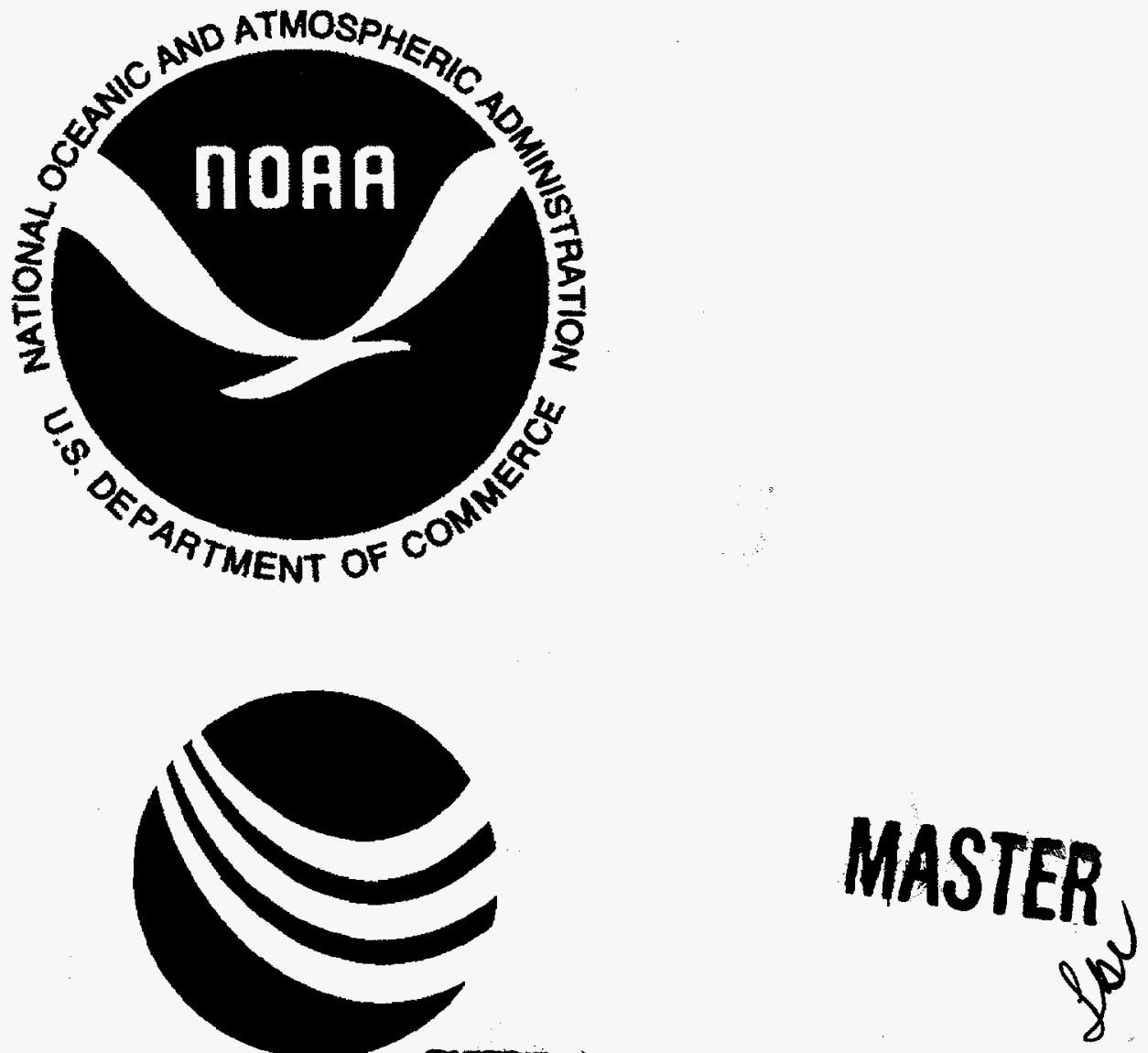

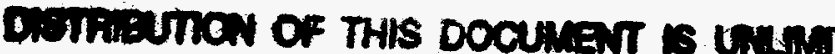

Carbon Dioxide Information Analysis Center

Oak Ridge National Laboratory

Environmental Sciences Division

Publication No. 4610 
This report has been reproduced directly from the best available copy.

Available to DOE and DOE contractors from the Office of Scientific and Technical Information, P.O. Box 62, Oak Ridge, TN 37831; prices available from (423) 576-8401, FTS 626-8401.

Available to the public from the National Technical Information Service, U.S. Department of Commerce, 5285 Port Royal Rd., Springfield, VA 22161.

This report was prepared as an account of work sponsored by an agency of the United States Government. Neither the United States Government nor any agency thereof, nor any of their employees, makes any warranty, express or implied, or assumes any legal liability or responsibility for the accuracy, completeness, or usefulness of any information, apparatus, product, or process disclosed, or represents that its use would not infringe privately owned rights. Reference herein to any specific commercial product, process, or service by trade name, trademark, manufacturer, or otherwise, does not necessarily constitute or imply its endorsement, recommendation, or favoring by the United States Government or any agency thereot. The views and opinions of authors expressed herein do not necessarily state or reflect those of the United States Government or any agency thereof. 


\section{DISCLAIMER}

Portions of this document may be illegible in electronic image products. Images are produced from the best available original document. 


\title{
DAILY SNOW DEPTH MEASUREMENTS FROM 195 STATIONS IN THE UNITED STATES
}

\author{
Contributed by \\ D. R. Easterling \\ P. Jamason \\ D. P. Bowman \\ P. Y. Hughes \\ E. H. Mason
}

National Oceanic and Atmospheric Administration

National Climatic Data Center

Asheville, North Carolina

Prepared by L. J. Allison

Carbon Dioxide Information Analysis Center

Oak Ridge National Laboratory

Oak Ridge, Tennessee

Environmental Sciences Division

Publication No. 4610

Date Published: February 1997

Prepared for the

Carbon Dioxide Research Program

Environmental Sciences Division

Office of Health and Environmental Research

Budget Activity Number KP 1204010

Prepared by the

Carbon Dioxide Information Analysis Center

OAK RIDGE NATIONAL LABORATORY

Oak Ridge, Tennessee 37831-6335

operated by

LOCKHEED MARTIN ENERGY RESEARCH CORP.

for the

U.S. DEPARTMENT OF ENERGY

under contract DE-AC05-96OR22464 


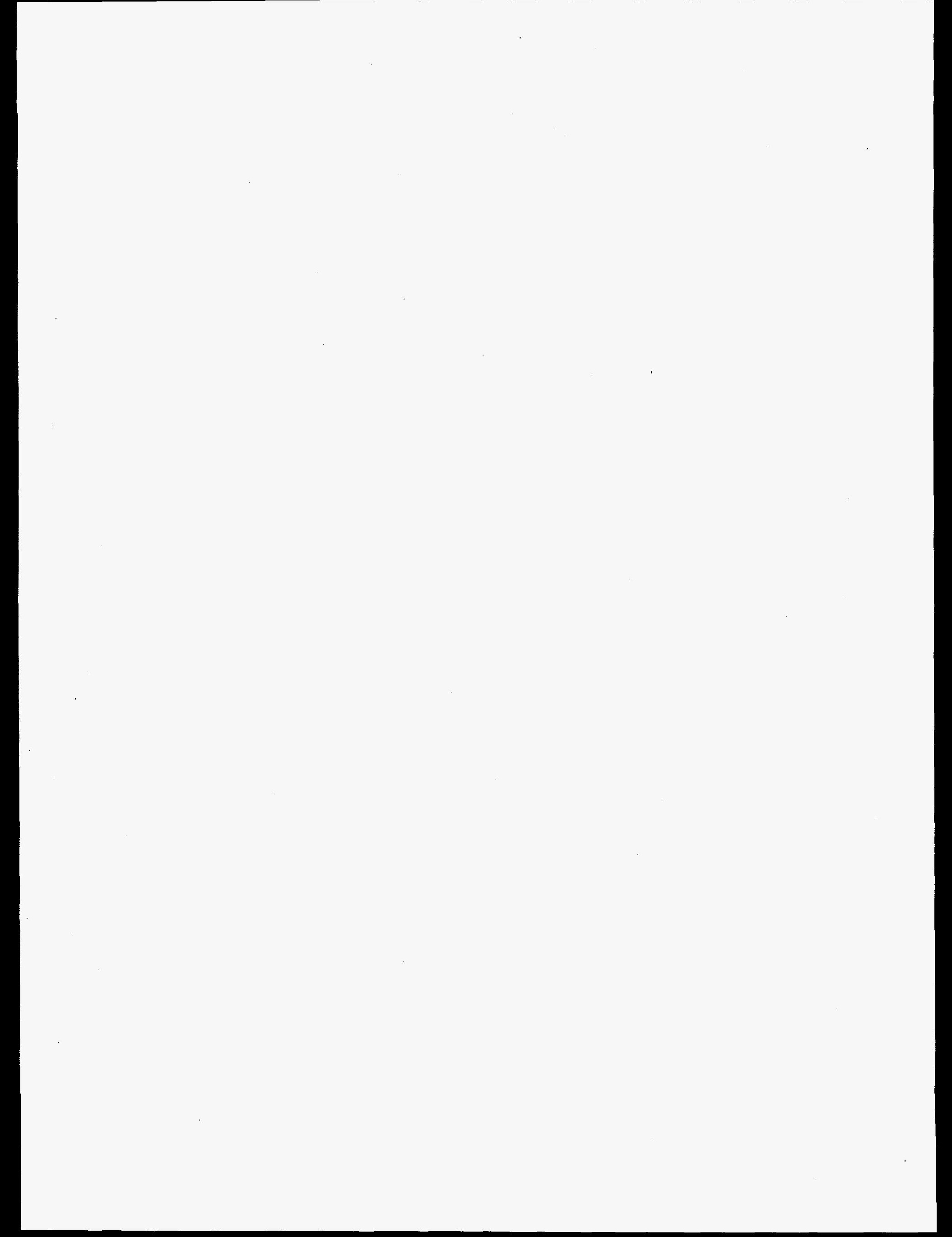




\section{CONTENTS}

LIST OF FIGURES $\ldots \ldots \ldots \ldots \ldots \ldots \ldots \ldots \ldots \ldots \ldots \ldots \ldots \ldots$

LIST OF TABLES $\ldots \ldots \ldots \ldots \ldots \ldots \ldots \ldots \ldots \ldots \ldots \ldots \ldots \ldots \ldots$ vii

ABSTRACT $\ldots \ldots \ldots \ldots \ldots \ldots \ldots \ldots \ldots \ldots \ldots \ldots \ldots \ldots \ldots \ldots$

PART 1: INFORMATION ABOUT THE DATA PACKAGE ......... 1

1. BACKGROUND INFORMATION $\ldots \ldots \ldots \ldots \ldots \ldots \ldots \ldots$

2. SOURCE AND SCOPE OF THE DATA $\ldots \ldots \ldots \ldots \ldots \ldots \ldots \ldots$

3. QUALITY ASSURANCE OF THE U.S. DAILY
SNOW DEPTH DATABASE $\ldots \ldots \ldots \ldots \ldots \ldots \ldots \ldots \ldots \ldots \ldots \ldots \ldots \ldots \ldots$

4. HOW TO OBTAIN THE DATA PACKAGE $\ldots \ldots \ldots \ldots \ldots \ldots \ldots$

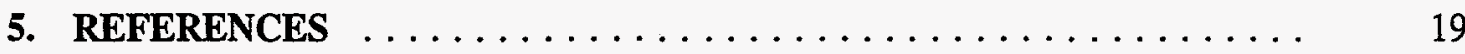

PART 2: CONTENT AND FORMAT OF DATA FILES . . . . . . . . 21

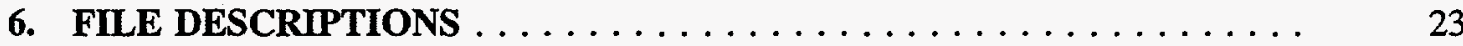

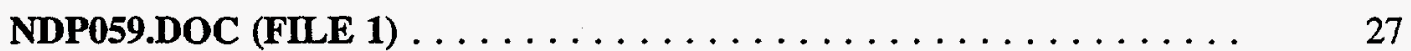

INVENT.FOR (FLLE 2) $\ldots \ldots \ldots \ldots \ldots \ldots \ldots \ldots \ldots$

DATA.FOR (FILE 3) $\ldots \ldots \ldots \ldots \ldots \ldots \ldots \ldots \ldots \ldots \ldots \ldots$

INVENT.SAS (FHE 4) $\ldots \ldots \ldots \ldots \ldots \ldots \ldots \ldots \ldots \ldots \ldots$

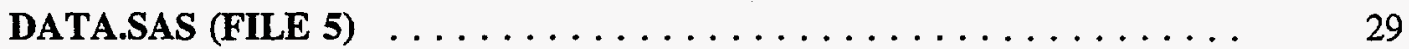

INVENT.DAT (FHE 6) $\ldots \ldots \ldots \ldots \ldots \ldots \ldots \ldots \ldots \ldots$

SNOWDPTH.DAT (FILE 7) $\ldots \ldots \ldots \ldots \ldots \ldots \ldots \ldots \ldots$

WEST.DAT (FILE 8) $\ldots \ldots \ldots \ldots \ldots \ldots \ldots \ldots \ldots \ldots \ldots$ 
HPLAINS.DAT (FILE 9) $\ldots \ldots \ldots \ldots \ldots \ldots \ldots \ldots \ldots \ldots \ldots$

SOUTH.DAT $($ FHE 10) $\ldots \ldots \ldots \ldots \ldots \ldots \ldots \ldots \ldots \ldots \ldots$

MUWEST.DAT (FLE 11) $\ldots \ldots \ldots \ldots \ldots \ldots \ldots \ldots \ldots$

SEAST.DAT (FILE 12) $\ldots \ldots \ldots \ldots \ldots \ldots \ldots \ldots \ldots \ldots \ldots \ldots$

NEAST.DAT (FILE 13) $\ldots \ldots \ldots \ldots \ldots \ldots \ldots \ldots \ldots \ldots \ldots$

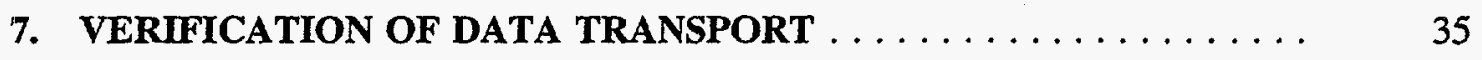

APPENDIX A: LIST OF STATE ABBREVIATIONS, NCDC STATE

CODES, AND STATE NAMES $\ldots \ldots \ldots \ldots \ldots \ldots \ldots$ A-1

APPENDIX B: REPRINT OF PERTINENT LITERATURE . ....

The Association between Extremes in North American Snow Cover Extent and United States Temperatures.

D. J. Leathers and D. A. Robinson. 1993. . . . . . . . . . . . . . B-3 


\section{LIST OF FIGURES}

Figure

$\underline{\text { Page }}$

1. Locations of the 195 stations in the U.S. daily snow depth database . . . . . 4

2. States included in each of the six regional snow depth data files $\ldots \ldots \ldots 26$ 


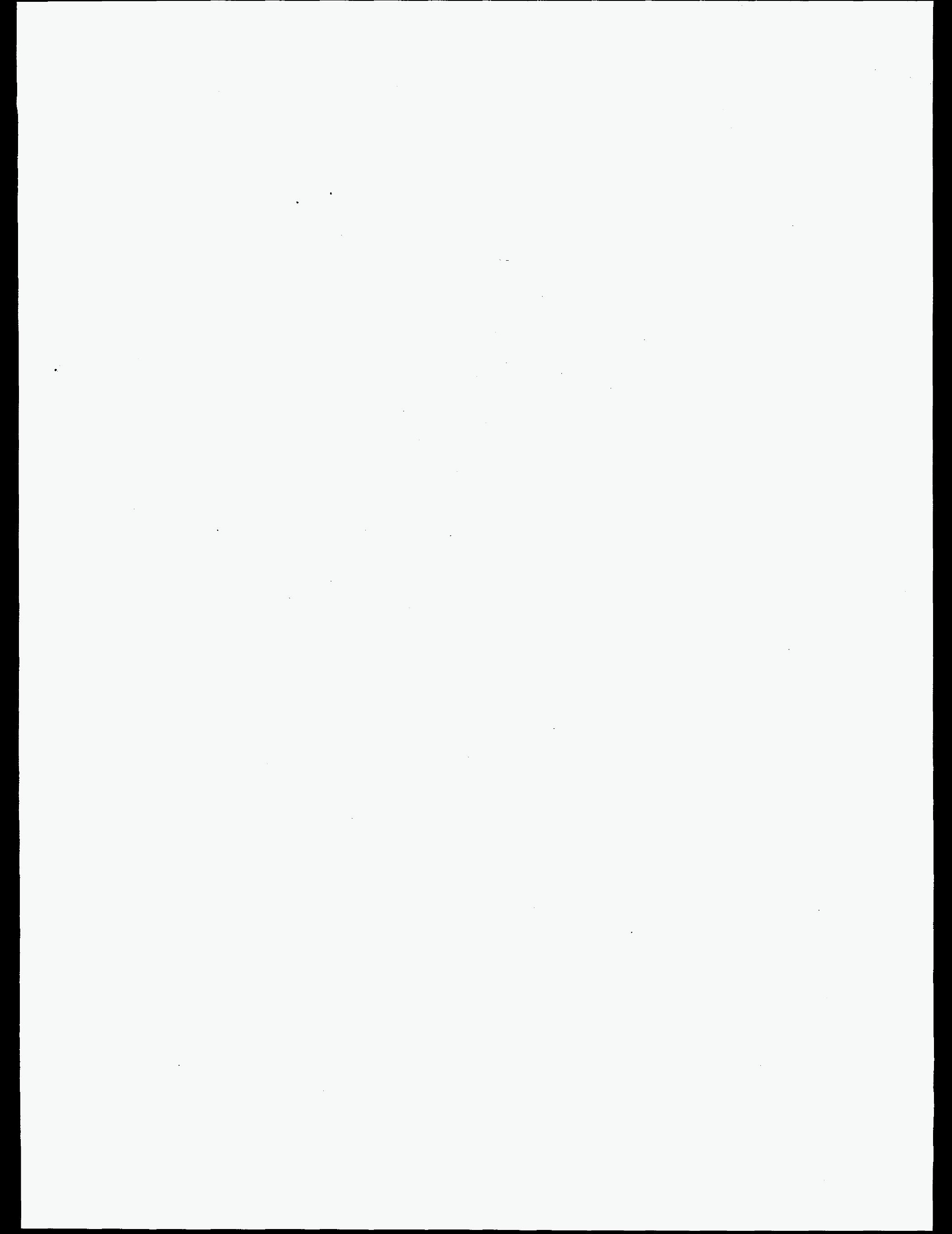




\section{LIST OF TABLES}

Table

$\underline{\text { Page }}$

1. Inventory of stations in the U.S. daily snow depth database $\ldots \ldots \ldots \ldots$

2. Size and format information for the NDP files .............. 24

3. Partial listing of the file invent.dat (File 6$) \ldots \ldots \ldots \ldots$

4. Partial listings of the snow depth files (Files 7-13) $\ldots \ldots \ldots \ldots \ldots \ldots$ 



\begin{abstract}
EASTERLING, D. R., P. JAMASON, D. BOWMAN, P. Y. HUGHES, AND

E. H. MASON. 1997. Daily Snow depth Measurements from 195 Stations in the United States. ORNL/CDIAC-95, NDP-059. Carbon Dioxide Information Analysis Center, Oak Ridge National Laboratory, Oak Ridge, Tennessee. 67 pp.
\end{abstract}

This document describes a database containing daily measurements of snow depth at 195 National Weather Service (NWS) first-order climatological stations in the United States. The data have been assembled and made available by the National Climatic Data Center (NCDC) in Asheville, North Carolina. The 195 stations encompass 388 unique sampling locations in 48 of the 50 states; no observations from Delaware or Hawaii are included in the database. Station selection criteria emphasized the quality and length of station records while seeking to provide a network with good geographic coverage. Snow depth at the 388 locations was measured once per day on ground open to the sky. The daily snow depth is the total depth of the snow on the ground at measurement time. The time period covered by the database is 1893-1992; however, not all station records encompass the complete period. While a station record ideally should contain daily data for at least the seven winter months (January through April and October through December), not all stations have complete records. Each logical record in the snow depth database contains one station's daily data values for a period of one month, including data source, measurement, and quality flags. The snow depth data have undergone extensive manual and automated quality assurance checks by NCDC and the Carbon Dioxide Information Analysis Center (CDIAC). These reviews involved examining the data for completeness, reasonableness, and accuracy, and included comparison of some data records with records in NCDC's Summary of the Day-First Order online database. Since the snow depth measurements have been taken at NWS first-order stations that have long periods of record, they should prove useful in monitoring climate change.

These data are available free of charge as a numeric data package (NDP) from CDIAC. The NDP consists of this document and 12 data files that are available via CDIAC's anonymous file transfer protocol (FTP) service, via the World Wide Web, and on 8-mm tape or quarter-inch tape cartridge. The total size of the database is $\sim 79,000 \mathrm{kB}$. 


\section{PART 1}

INFORMATION ABOUT THE DATA PACKAGE 


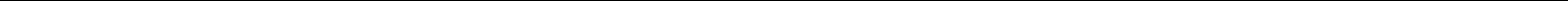




\section{BACKGROUND INFORMATION}

Daily snow depth records from 195 stations in the United States have been assembled into a database by the National Climatic Data Center (NCDC). Since the measurements have been taken at National Weather Service (NWS) principal climatological stations that have long periods of record, the snow depth data should prove useful in monitoring climate change. The continued accumulation of anthropogenic greenhouse gases in the atmosphere over the past century has commonly been linked to an increase in global mean temperature (IPCC 1996). This increase in temperature may be indicative of regional changes that could be reflected in changes in snowfall and in the depth, extent, and duration of snow cover. It has been observed that the annual snow cover extent over North America in the last 19 years has decreased relative to a $0.93^{\circ} \mathrm{C}$ increase in temperature in this region (Karl et al. 1993). Since 1988, the snow cover extent over the Northem Hemisphere has been consistently below the 21-year average for the period 1974-1994 (Nicholls et al. 1996). Other studies have examined the relationship between the snow cover extent in North America and United States winter temperatures (Leathers and Robinson 1993) and the trends and variability in snowfall and total precipitation across the United States and Canada for 1951-1990 (Groisman and Easterling 1994). The U.S. snow depth database should be useful in continuing to monitor such changes over the United States. It also enables analysis of snow cover changes for the period prior to the advent of accurate satellite-derived estimates, which began in 1972 (Leathers and Robinson 1993).

\section{SOURCE AND SCOPE OF THE DATA}

The 195 stations in the U.S. daily snow depth database are a subset of the NWS's principal climatological stations (often referred to as the "first-order" network). They overlap with many of the stations included in the U.S. historical sunshine and cloud cover database (Steurer and Karl 1991) and with the 223 U.S. primary stations included in the North American network used by Groisman and Easterling (1994) to study trends and variability in precipitation and snowfall for 1951-1990. These 195 stations encompass 388 unique sampling locations in 48 states; no observations from Delaware or Hawaii are included in the database. A map showing the locations of the 195 snow depth stations is presented in Fig. 1.

Station selection criteria emphasized the quality and length of station records while seeking to provide a network with good geographic coverage. Many sampling stations were relocated from a city to an airport or other remote location or changed elevation during the course of the data record, resulting in multiple locations for these stations. Measurements were often taken at both stations for a period of time to provide some overlap in the data record. Snow depth was measured once per day on ground open to the sky. The daily snow depth is the total depth of the snow (old and new) on the ground at measurement time. An inventory of the 195 snow depth stations-including the state abbreviation, station name, NCDC Cooperative Network Index station number, Weather Bureau Army Navy (WBAN) station number, latitude, longitude, elevation above sea level, period of station record, and a flag denoting the completeness of the station record-is given in Table 1. The multiple sampling locations associated with some of the stations are also listed in this table. The snow depth station inventory was compiled from several sources; its development is described in detail in Part 2 of this document.

The snow depth data were compiled by NCDC in two phases: data collected before 1948 were input from manuscript forms, while data recorded from 1948-1992 were extracted 
from digital tape archives. Initially, only months containing nonzero snow depth values were to be digitized; however, this created an ambiguity with months that were reported as "no snow" (zero snow depth) versus months missing from the data record. Thus, NCDC conducted a manual review of all the manuscript forms for the period before 1948 to resolve the issue of whether the data being digitized were indeed truly missing. Gaps in the station records in some cases result from missing manuscripts.

The time period covered by the snow depth database is 1893-1992. In general, snowfall occurs between October and April; however, several stations may receive snowfall as early as September or as late as July. Ideally, each full year of a station's record should contain at least seven months of "nonmissing" data (January through April and October through December, hereafter referred to as the winter months). However, many stations do not have all seven winter months in all years of the station record. In addition, only 72 stations span the 100-year recording period, and many of these station records are missing one or more months, or even years, of data (see Table 1). In order to characterize the completeness and usefulness of station records, close examination of the data was required. For example, some stations that have seven months of data in a given year often have one or more winter months missing (e.g., Station 132367, Dubuque, Iowa, has nine months of data in 1981 but is missing the winter months of November and December). Of the 388 sampling locations, 151 (38.9\%) have complete records for October-April or are missing only one or two months of data in the station record. Seventy locations (18.0\%) have several years in the data record that are missing one to three months of data, 92 locations $(23.7 \%$ ) have many years that are missing at least one and usually many months of data, and 70 locations (18.0\%) are missing one or more years of data in the station record. Five stations have records of only one year in length; three of these records have fewer than seven winter months of data.

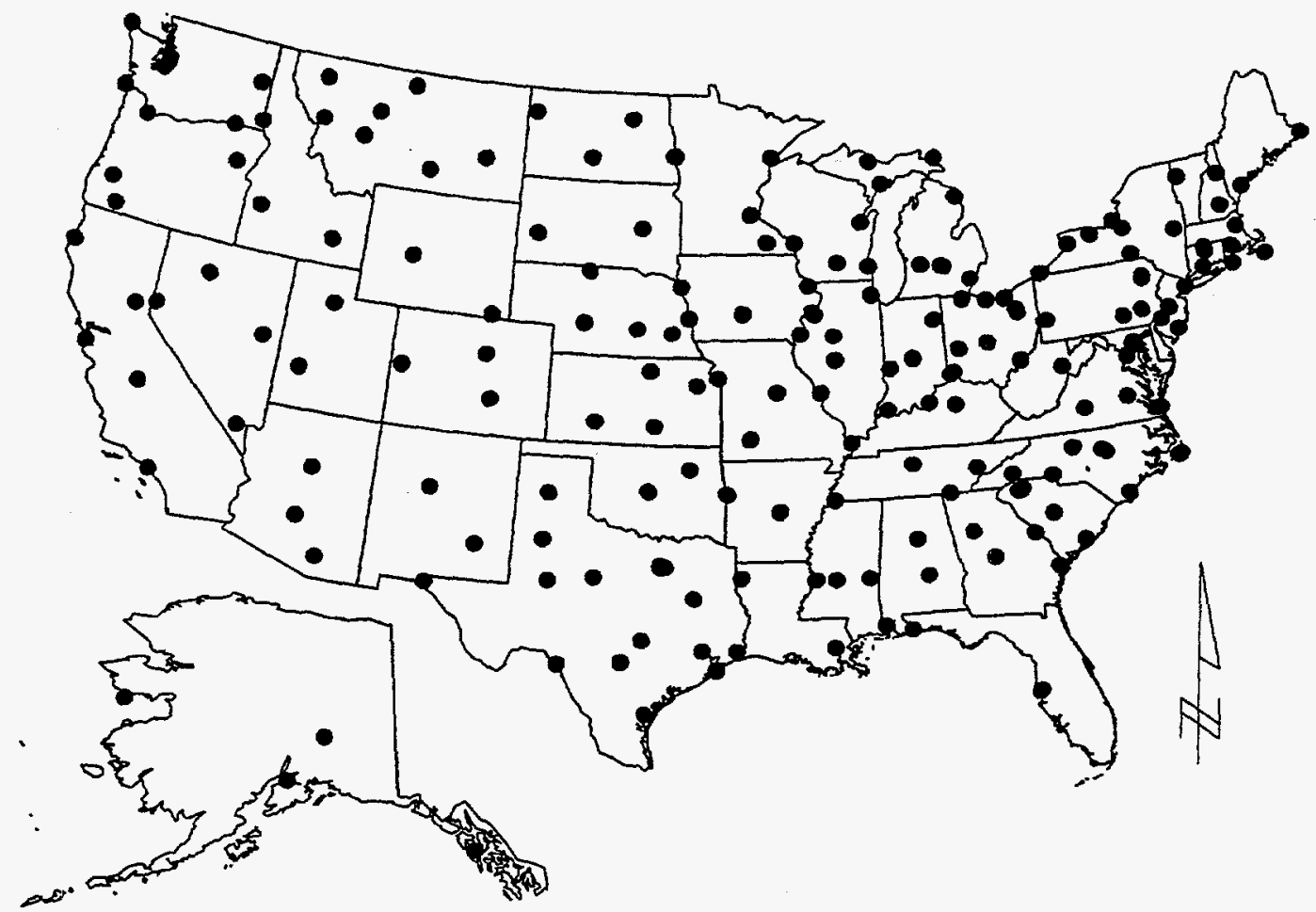

Fig. 1. Locations of the 195 stations in the U.S. daily snow depth database. 
Table 1. Inventory of stations in the U.S. daily snow depth database.

\begin{tabular}{|c|c|c|c|c|c|c|c|c|}
\hline State $^{a}$ & $\begin{array}{l}\text { Station } \\
\text { name }\end{array}$ & $\begin{array}{c}\mathrm{NCDC}^{b} \\
\text { station no. }\end{array}$ & $\begin{array}{l}\text { WBAN }^{c} \\
\text { sta. no. }\end{array}$ & $\begin{array}{l}\text { Lat. }^{d} \\
\left({ }^{\circ} \mathrm{N}\right)\end{array}$ & $\begin{array}{l}\text { Long. }{ }^{d} \\
\left({ }^{\circ} \mathrm{W}\right)\end{array}$ & $\begin{array}{l}\text { Elev. }^{e} \\
(\mathrm{~m})\end{array}$ & $\begin{array}{l}\text { Period } \\
\text { of Record }\end{array}$ & $\begin{array}{c}\text { Record } \\
\text { Completeness }^{f}\end{array}$ \\
\hline \multirow[t]{13}{*}{ AK } & \multirow[t]{3}{*}{ Anchorage } & 50ANCC & 99999 & -99.99 & -99.99 & -99.99 & $1923-1943$ & $\mathrm{E}$ \\
\hline & & 500285 & 26409 & 61.22 & 149.83 & 43.00 & $1943-1953$ & B \\
\hline & & 500280 & 26451 & 61.17 & 150.02 & 35.00 & 1953-1992 & B \\
\hline & \multirow[t]{2}{*}{ Fairbanks } & 50FAIC & 99999 & 64.83 & 147.72 & 148.00 & 1929-1942 & B \\
\hline & & 502968 & 26411 & 64.82 & 147.87 & 133.00 & 1942-1992 & B \\
\hline & \multirow[t]{4}{*}{ Juneau } & 504094 & 25324 & 58.30 & 134.40 & 24.00 & $1917-1921$ & $A$ \\
\hline & & 504094 & 25324 & 58.30 & 134.40 & 58.00 & $1922-1930$ & B \\
\hline & & 504094 & 25324 & 58.30 & 134.40 & 40.00 & $1931-1943$ & B \\
\hline & & 504100 & 25309 & 58.37 & 134.58 & 3.00 & 1943-1992 & B \\
\hline & \multirow[t]{4}{*}{ Nome } & 500MEV & 99999 & 64.48 & 165.35 & 10.00 & 1930 & $\mathrm{G}$ \\
\hline & & 50OMEV & 99999 & 64.48 & 165.40 & 4.00 & $1931-1945$ & B \\
\hline & & 50OMEV & 99999 & 64.50 & 165.42 & 5.00 & 1946 & $\mathrm{G}$ \\
\hline & & 506496 & 26617 & 64.50 & 165.43 & 4.00 & 1946-1992 & B \\
\hline \multirow[t]{6}{*}{$\mathrm{AL}$} & \multirow[t]{2}{*}{ Birmingham } & 010836 & 93869 & 33.52 & 86.83 & 212.00 & $1904-1953$ & $\mathrm{C}$ \\
\hline & & 010831 & 13876 & 33.57 & 86.75 & 189.00 & $1944-1992$ & A \\
\hline & \multirow[t]{2}{*}{ Mobile } & 015483 & 93855 & 30.68 & 88.03 & 36.00 & $1893-1965$ & $\mathrm{C}$ \\
\hline & & 015478 & 13894 & 30.68 & 88.25 & 64.00 & $1948-1992$ & $\mathrm{~A}$ \\
\hline & \multirow[t]{2}{*}{ Montgomery } & 015555 & 93856 & 32.38 & 86.30 & 61.00 & $1893-1954$ & $\mathrm{C}$ \\
\hline & & 015550 & 13895 & 32.30 & 86.40 & 67.00 & 1948-1992 & A \\
\hline \multirow[t]{4}{*}{ AR } & \multirow[t]{2}{*}{ Fort Smith } & 03FSMC & 93946 & 35.37 & 94.40 & 137.00 & $1893-1945$ & $\mathrm{D}$ \\
\hline & & 032574 & 13964 & 35.33 & 94.37 & 137.00 & 1945-1992 & B \\
\hline & \multirow[t]{2}{*}{ Little Rock } & 03LITC & 93930 & 34.75 & 92.27 & 99.00 & $1893-1942$ & $\mathrm{C}$ \\
\hline & & 034248 & 13963 & 34.73 & 92.22 & 84.00 & 1940-1992 & $\mathrm{C}$ \\
\hline \multirow[t]{6}{*}{$\mathrm{AZ}$} & \multirow[t]{2}{*}{ Flagstaff } & 023007 & 23166 & 35.20 & 111.67 & 2104.00 & 1899-1950 & $\mathrm{D}$ \\
\hline & & 023010 & 03103 & 35.13 & 111.67 & 2132.00 & 1950-1992 & $\mathrm{C}$ \\
\hline & \multirow[t]{2}{*}{ Phoenix } & 026486 & 93140 & 33.45 & 112.07 & 335.00 & $1937-1968$ & $B$ \\
\hline & & 026481 & 23183 & 33.43 & 112.02 & 338.00 & $1948-1992$ & $\mathrm{~A}$ \\
\hline & \multirow[t]{2}{*}{ Tucson } & 028815 & 23193 & 32.25 & 110.95 & 745.00 & $1928-1992$ & $\mathrm{E}$ \\
\hline & & 028820 & 23160 & 32.13 & 110.93 & 788.00 & 1941-1992 & A \\
\hline \multirow[t]{8}{*}{$\mathrm{CA}$} & Blue Canyon & 040897 & 23225 & 39.28 & 120.70 & 1610.00 & 1939-1990 & $\mathrm{E}$ \\
\hline & Eureka & 042910 & 24213 & 40.80 & 124.17 & 18.00 & $1906-1992$ & $\bar{C}$ \\
\hline & \multirow[t]{2}{*}{ Fresno } & 04FATC & 99999 & 36.73 & 119.78 & 87.00 & $1929-1939^{8}$ & $\mathrm{C}$ \\
\hline & & 043257 & 93193 & 36.77 & 119.72 & 100.00 & $1949-1992^{8}$ & A \\
\hline & \multirow[t]{2}{*}{ Los Angeles } & 045115 & 93134 & 34.05 & 118.23 & 78.00 & $1930-1992$ & $E$ \\
\hline & & 045114 & 23174 & 33.93 & 118.38 & 30.00 & 1948-1992 & A \\
\hline & \multirow[t]{2}{*}{ San Francisco } & 047772 & 23272 & 37.77 & 122.43 & 22.00 & 1932-1992 & $\mathrm{E}$ \\
\hline & & 047769 & 23234 & 37.62 & 122.38 & 2.00 & 1948-1992 & $\bar{A}$ \\
\hline
\end{tabular}


Table 1 (continued)

\begin{tabular}{|c|c|c|c|c|c|c|c|c|}
\hline State $^{a}$ & $\begin{array}{l}\text { Station } \\
\text { name }\end{array}$ & $\begin{array}{c}\mathrm{NCDC}^{b} \\
\text { station no. }\end{array}$ & $\begin{array}{l}\text { WBAN }^{c} \\
\text { sta. no. }\end{array}$ & $\begin{array}{l}\text { Lat. }^{d} \\
\left({ }^{\circ} \mathrm{N}\right)\end{array}$ & $\begin{array}{l}\text { Long. }{ }^{d} \\
\left({ }^{\circ} \mathrm{W}\right)\end{array}$ & $\begin{array}{l}\text { Elev.e } \\
(\mathrm{m})\end{array}$ & $\begin{array}{l}\text { Period } \\
\text { of Record }\end{array}$ & $\begin{array}{c}\text { Record } \\
\text { Completeness }\end{array}$ \\
\hline \multirow[t]{7}{*}{$\mathrm{CO}$} & Denver & 052225 & 93002 & 39.75 & 105.00 & 1591.00 & $1893-1948$ & $\mathrm{C}$ \\
\hline & & 052220 & 23062 & 39.77 & 104.87 & 1612.00 & $1945-1992$ & A \\
\hline & Grand Junction & $05 \mathrm{GJCT}$ & 93031 & 39.07 & 108.57 & 1398.00 & $1899-1946$ & $\mathrm{D}$ \\
\hline & & 053488 & 23066 & 39.10 & 108.55 & 1478.00 & 1946-1992 & B \\
\hline & Pueblo & 05PUBC & 93022 & 38.30 & 104.60 & 1423.00 & $1893-1940$ & $\mathrm{D}$ \\
\hline & & 056738 & 23068 & 38.23 & 104.63 & 1463.00 & 1940-1954 & $\mathrm{D}$ \\
\hline & & 056740 & 93058 & 38.28 & 104.52 & 1420.00 & 1954-1992 & B \\
\hline \multirow[t]{6}{*}{$\mathrm{CT}$} & Hartford & 063451 & 14752 & 41.73 & 72.65 & 5.00 & $1940-1954$ & C \\
\hline & & 063456 & 14740 & 41.93 & 72.68 & 49.00 & $1954-1992$ & A \\
\hline & New Haven & 065266 & 94772 & 41.30 & 72.93 & 37.00 & $1893-1918$ & $\mathrm{D}$ \\
\hline & & 065266 & 94772 & 41.30 & 72.93 & 23.00 & 1919-1943 & $\mathrm{D}$ \\
\hline & & 065273 & 14758 & 41.27 & 72.88 & 7.00 & $1939-1969$ & $\mathrm{D}$ \\
\hline & Windsor Locks & 06BDLC & 94760 & 41.77 & 72.68 & 18.00 & 1904-1940 & $D$ \\
\hline \multirow[t]{4}{*}{ FL } & Pensacola & 087002 & 93859 & 30.42 & 87.22 & 4.00 & $1895-1963$ & $\mathrm{C}$ \\
\hline & & 086997 & 13899 & 30.47 & 87.20 & 34.00 & $1943-1992$ & $\mathrm{~B}$ \\
\hline & T'ampa & 08TPAC & 99999 & 27.95 & 82.45 & 6.00 & 1940 & $\mathrm{~F}$ \\
\hline & & 088788 & 12842 & 27.97 & 82.53 & 6.00 & $1948-1992$ & $\mathrm{~A}$ \\
\hline \multirow[t]{8}{*}{ GA } & Atlanta & 090456 & 93847 & 33.75 & 84.38 & 321.00 & $1896-1954$ & $\mathrm{D}$ \\
\hline & & 090451 & 13874 & 33.65 & 84.43 & 308.00 & $1935-1992$ & $\mathrm{C}$ \\
\hline & Augusta & 090500 & 93848 & 33.47 & 81.97 & 41.00 & $1893-1949$ & $\mathrm{D}$ \\
\hline & & 090495 & 13875 & 33.37 & 81.97 & 45.00 & $1948-1992$ & $\mathrm{~A}$ \\
\hline & Macon & 095447 & 93853 & 32.83 & 83.63 & 101.00 & $1899-1948$ & $\mathrm{E}$ \\
\hline & & 095443 & 03813 & 32.70 & 83.65 & 108.00 & $1948-1992$ & $\bar{A}$ \\
\hline & Savannah & O9SAVC & 93860 & 32.08 & 81.08 & 12.00 & $1893-1945^{8}$ & $\mathrm{C}$ \\
\hline & & 097847 & 93802 & 32.13 & 81.20 & 14.00 & $1948-1992^{8}$ & A \\
\hline \multirow[t]{12}{*}{ IA } & Burlington & 131063 & 14931 & 40.78 & 91.12 & 214.00 & $1942-1947$ & $\mathrm{D}$ \\
\hline & & 131063 & 14931 & 40.78 & 91.12 & 213.00 & $1947-1979$ & $\mathrm{C}$ \\
\hline & Davenport & 132069 & 14932 & 41.52 & 90.58 & 189.00 & $1893-1896$ & $\mathrm{D}$ \\
\hline & & 132069 & 14932 & 41.52 & 90.57 & 185.00 & $1897-1931$ & $D$ \\
\hline & & 132069 & 14932 & 41.52 & 90.57 & 202.00 & $1932-1933$ & $\mathrm{C}$ \\
\hline & & 132069 & 14932 & 41.52 & 90.57 & 176.00 & $1934-1943$ & $\mathrm{D}$ \\
\hline & Des Moines & 132208 & 14967 & 41.58 & 93.62 & 246.00 & $1893-1973$ & $\mathrm{E}$ \\
\hline & & 132203 & 14933 & 41.53 & 93.65 & 286.00 & $1938-1992$ & $\mathrm{~B}$ \\
\hline & Dubuque & 132369 & 14934 & 42.50 & 90.67 & 195.00 & 1893-1951 & $\mathrm{D}$ \\
\hline & & 132367 & 94908 & 42.40 & 90.70 & 324.00 & $1951-1992$ & $\mathrm{C}$ \\
\hline & Sioux City & 13SIUC & 14987 & 42.50 & 96.40 & 338.00 & $1893-1941$ & $\mathrm{D}$ \\
\hline & & 137708 & 14943 & 42.40 & 96.38 & 336.00 & $1940-1992$ & $\mathrm{C}$ \\
\hline
\end{tabular}


Table 1 (continued)

\begin{tabular}{|c|c|c|c|c|c|c|c|c|}
\hline State ${ }^{a}$ & $\begin{array}{l}\text { Station } \\
\text { name }\end{array}$ & $\begin{array}{c}\mathrm{NCDC}^{b} \\
\text { station no. }\end{array}$ & $\begin{array}{l}\text { WBAN }^{c} \\
\text { sta. no. }\end{array}$ & $\begin{array}{l}\text { Lat. }^{d} \\
\left({ }^{\circ} \mathrm{N}\right)\end{array}$ & $\begin{array}{l}\text { Long. } \\
\text { ( W) }\end{array}$ & $\begin{array}{l}\text { Elev. }{ }^{e} \\
(\mathrm{~m})\end{array}$ & $\begin{array}{l}\text { Period } \\
\text { of Record }\end{array}$ & $\begin{array}{c}\text { Record } \\
\text { Completeness }{ }^{f}\end{array}$ \\
\hline \multirow[t]{6}{*}{ ID } & \multirow[t]{2}{*}{ Boise } & 10BOIC & 99999 & 43.62 & 116.20 & 827.00 & $1898-1939$ & D \\
\hline & & 101022 & 24131 & 43.57 & 116.22 & 865.00 & 1938-1992 & $\mathrm{C}$ \\
\hline & \multirow[t]{2}{*}{ Lewiston } & 105236 & 94154 & 46.42 & 117.03 & 264.00 & $1900-1947$ & $\mathrm{D}$ \\
\hline & & 105241 & 24149 & 46.38 & 117.02 & 438.00 & $1947-1992$ & $\mathrm{~B}$ \\
\hline & \multirow[t]{2}{*}{ Pocatello } & 10PIHC & 99999 & 42.87 & 112.48 & 1362.00 & $1899-1940$ & $\mathrm{D}$ \\
\hline & & 107211 & 24156 & 42.92 & 112.60 & 1358.00 & $1938-1992$ & $\mathrm{C}$ \\
\hline \multirow[t]{9}{*}{ II } & Cairo & 111166 & 93809 & 37.00 & 89.17 & 95.00 & 1893-1992 & $\mathrm{D}$ \\
\hline & \multirow[t]{3}{*}{ Chicago } & 111582 & 14881 & 41.88 & 87.63 & 181.00 & 1893-1925 & $\mathrm{C}$ \\
\hline & & 111572 & 14892 & 41.78 & 87.60 & 181.00 & $1916-1948^{8}$ & $\mathrm{E}$ \\
\hline & & 111549 & 94846 & 41.98 & 87.90 & 205.00 & $1958-1992^{8}$ & A \\
\hline & Moline & 115751 & 14923 & 41.45 & 90.50 & 177.00 & 1931-1992 & $\mathrm{D}$ \\
\hline & \multirow[t]{2}{*}{ Peoria } & 11PIAC & 14874 & 40.72 & 89.60 & 183.00 & $1905-1943$ & D \\
\hline & & 116711 & 14842 & 40.67 & 89.68 & 198.00 & 1935-1992 & B \\
\hline & \multirow[t]{2}{*}{ Springfield } & 118179 & 93822 & 39.85 & 89.68 & 181.00 & 1935-1992 & B \\
\hline & & 118184 & 93896 & 39.80 & 89.65 & 182.00 & $1893-1950$ & $\mathrm{D}$ \\
\hline \multirow[t]{10}{*}{$\mathbb{I N}$} & \multirow[t]{2}{*}{ Evansville } & $12 \mathrm{EVVC}$ & 99999 & 37.97 & 87.55 & 118.00 & $1897-1940$ & $D$ \\
\hline & & 122738 & 93817 & 38.05 & 87.53 & 116.00 & 1941-1992 & A \\
\hline & \multirow[t]{2}{*}{ Fort Wayne } & 123024 & 99999 & 41.08 & 85.17 & 237.00 & $1911-1946$ & $\mathrm{D}$ \\
\hline & & 123037 & 14827 & 41.00 & 85.20 & 243.00 & $1942-1992$ & B \\
\hline & \multirow[t]{2}{*}{ Indianapolis } & 124264 & 93892 & 39.77 & 86.17 & 219.00 & 1893-1954 & $\mathrm{D}$ \\
\hline & & 124259 & 93819 & 39.73 & 86.27 & 241.00 & $1940-1992$ & B \\
\hline & \multirow[t]{4}{*}{ Terre Haute } & 12HUFC & 99999 & 39.48 & 87.40 & 152.00 & $1912-1935$ & A \\
\hline & & 12HUFC & 99999 & 39.48 & 87.40 & 153.00 & 1935-1947 & $\mathrm{C}$ \\
\hline & & 128725 & 93823 & 39.45 & 87.30 & 177.00 & $1947-1948$ & B \\
\hline & & 128725 & 93823 & 39.45 & 87.30 & 181.00 & $1948-1954$ & B \\
\hline \multirow[t]{9}{*}{$\mathrm{KS}$} & \multirow[t]{2}{*}{ Concordia } & 141769 & 99999 & 39.57 & 97.67 & 419.00 & 1893-1962 & $\mathrm{E}$ \\
\hline & & 141767 & 13984 & 39.55 & 97.65 & 448.00 & $1962-1992$ & A \\
\hline & \multirow[t]{2}{*}{ Dodge City } & 14DDCC & 93016 & 37.75 & 100.02 & 769.00 & 1893-1942 & $\mathrm{D}$ \\
\hline & & 142164 & 13985 & 37.77 & 99.97 & 787.00 & 1942-1992 & $\mathrm{B}$ \\
\hline & \multirow[t]{2}{*}{ Topeka } & 148172 & 93964 & 39.05 & 95.67 & 282.00 & $1899-1950$ & $\mathrm{D}$ \\
\hline & & 148167 & 13996 & 39.07 & 95.63 & 267.00 & 1946-1992 & B \\
\hline & \multirow[t]{3}{*}{ Wichita } & 14ICTC & 93982 & 37.68 & 97.33 & 396.00 & $1893-1940$ & $\mathrm{D}$ \\
\hline & & 148828 & 13998 & 37.63 & 97.27 & 418.00 & $1940-1953$ & $\mathrm{D}$ \\
\hline & & 148830 & 03928 & 37.65 & 97.43 & 403.00 & 1953-1992 & B \\
\hline \multirow[t]{4}{*}{ KY } & Cincinnati/ & 151855 & 93814 & 39.07 & 84.67 & 270.00 & 1948-1992 & A \\
\hline & \multicolumn{8}{|c|}{ Northern Kentucky } \\
\hline & Lexington & 15LEXC & 99999 & 38.03 & 84.55 & 306.00 & $1893-1944$ & D \\
\hline & & 154746 & 93820 & 38.03 & 84.60 & 295.00 & 1944-1992 & B \\
\hline
\end{tabular}


Table 1 (continued)

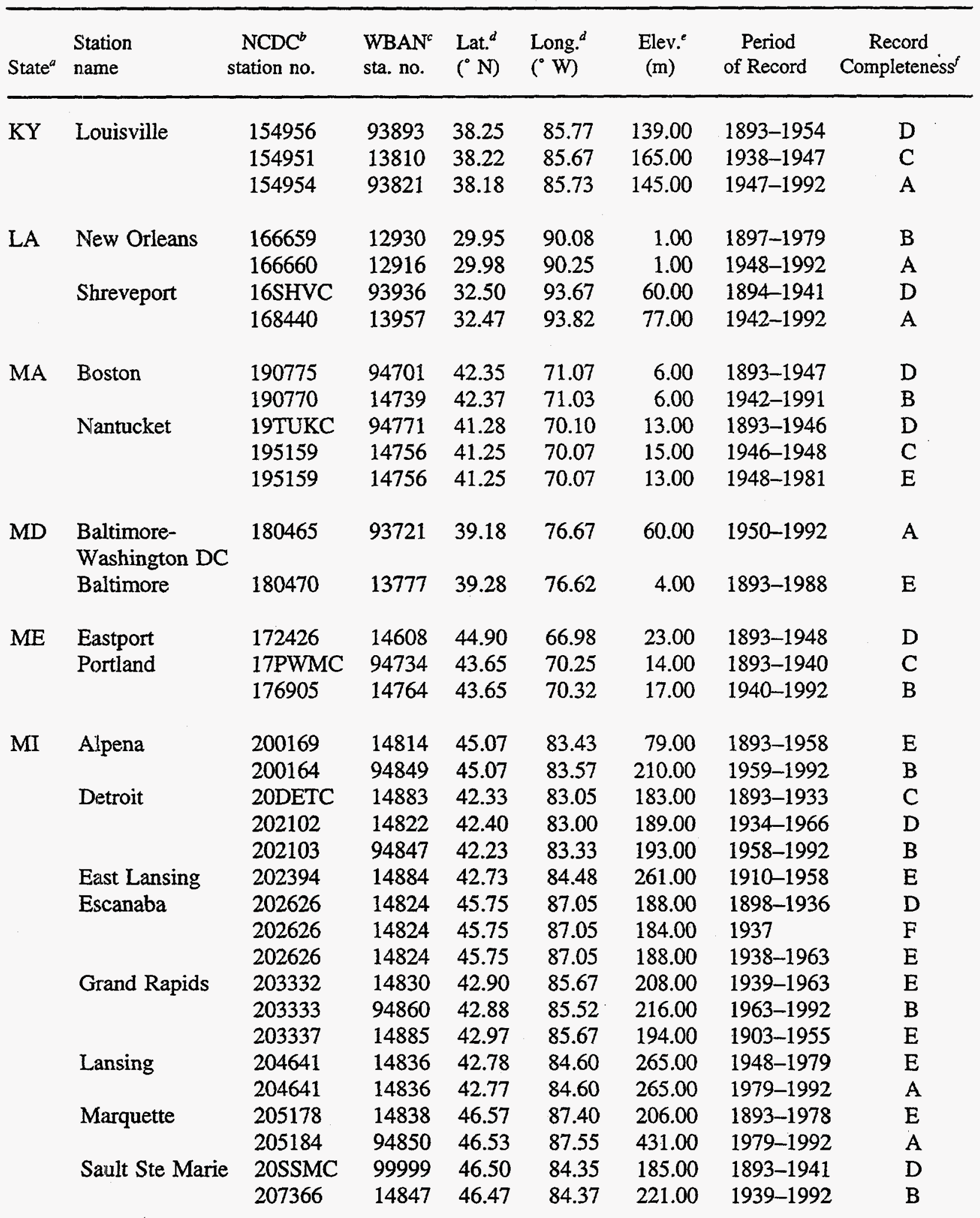


Table 1 (continued)

\begin{tabular}{|c|c|c|c|c|c|c|c|c|}
\hline State ${ }^{a}$ & $\begin{array}{l}\text { Station } \\
\text { name }\end{array}$ & $\begin{array}{c}\text { NCDC }^{b} \\
\text { station no. }\end{array}$ & $\begin{array}{l}\text { WBAN }^{c} \\
\text { sta. no. }\end{array}$ & $\begin{array}{l}\text { Lat. }^{d} \\
\left({ }^{\circ} \mathrm{N}\right)\end{array}$ & $\begin{array}{l}\text { Long. } \\
\text { ( } \mathrm{W})\end{array}$ & $\begin{array}{l}\text { Elev. }{ }^{e} \\
(\mathrm{~m})\end{array}$ & $\begin{array}{l}\text { Period } \\
\text { of Record }\end{array}$ & $\begin{array}{c}\text { Record } \\
\text { Completeness }\end{array}$ \\
\hline \multirow[t]{6}{*}{$\mathrm{MN}$} & Duluth & 212253 & 14959 & 46.78 & 92.10 & 354.00 & $1893-1959$ & $E$ \\
\hline & & 212248 & 14913 & 46.83 & 92.18 & 435.00 & 1945-1992 & B \\
\hline & Minneapolis & 21MSPC & 14961 & 44.98 & 93.30 & 256.00 & 1899-1938 & $\mathrm{C}$ \\
\hline & & 215435 & 14922 & 44.88 & 93.22 & 254.00 & 1938-1992 & B \\
\hline & Moorhead & 215589 & 99999 & 46.87 & 96.73 & 275.00 & $1893-1942$ & $\mathrm{C}$ \\
\hline & Rochester & 217004 & 14925 & 43.92 & 92.50 & 395.00 & 1939-1992 & D \\
\hline \multirow[t]{10}{*}{ MO } & Columbia & 231795 & 93958 & 38.95 & 92.33 & 223.00 & $1893-1950$ & $\mathrm{C}$ \\
\hline & & 231790 & 13983 & 38.97 & 92.37 & 237.00 & $1940-1969$ & $\mathrm{E}$ \\
\hline & & 231791 & 03945 & 38.82 & 92.22 & 270.00 & 1969-1992 & B \\
\hline & Kansas City & $23 \mathrm{MKCC}$ & 93972 & 39.08 & 94.58 & 245.00 & 1893-1933 & $\mathrm{C}$ \\
\hline & & 234359 & 13988 & 39.12 & 94.60 & 226.00 & 1934-1972 & $\mathrm{C}$ \\
\hline & & 234358 & 03947 & 39.32 & 94.72 & 297.00 & 1972-1992 & B \\
\hline & Springfield & 23SGFC & 93981 & 37.20 & 93.30 & 396.00 & $1893-1940$ & $D$ \\
\hline & & 237976 & 13995 & 37.23 & 93.38 & 387.00 & 1940-1992 & B \\
\hline & St. Louis & 237460 & 93963 & 38.63 & 90.20 & 142.00 & $1893-1968$ & $\mathrm{D}$ \\
\hline & & 237455 & 13994 & 38.75 & 90.37 & 163.00 & 1939-1992 & $\mathrm{C}$ \\
\hline \multirow[t]{7}{*}{ MS } & Jackson & 224462 & 99999 & 32.28 & 90.18 & 62.00 & 1915-1939 & $\mathrm{E}$ \\
\hline & & 224467 & 13956 & 32.33 & 90.22 & 93.00 & $1940-1963$ & B \\
\hline & & 224472 & 03940 & 32.32 & 90.08 & 101.00 & 1963-1992 & $\mathrm{B}$ \\
\hline & Meridian & 225772 & 13854 & 32.35 & 88.67 & 116.00 & $1893-1948$ & $\mathrm{C}$ \\
\hline & & 225776 & 13865 & 32.33 & 88.75 & 88.00 & $1948-1992$ & A \\
\hline & Vicksburg & 229220 & 93917 & 32.35 & 90.88 & 75.00 & 1893-1936 & $E$ \\
\hline & & 229220 & 93917 & 32.35 & 90.88 & 90.00 & $1937-1967$ & $E$ \\
\hline \multirow[t]{13}{*}{$\mathrm{MT}$} & Billings & 240807 & 24033 & 45.80 & 108.53 & 1088.00 & 1939-1992 & B \\
\hline & Great Falls & 243749 & 99999 & 47.52 & 111.30 & 1021.00 & $1938-1956$ & $E$ \\
\hline & & 243751 & 24143 & 47.48 & 111.37 & 1117.00 & 1942-1992 & B \\
\hline & Havre & 243994 & 24035 & 48.57 & 109.67 & 758.00 & $1893-1961$ & D \\
\hline & & 243996 & 94012 & 48.55 & 109.77 & 788.00 & $1961-1992$ & C \\
\hline & Helena & 244057 & 94104 & 46.58 & 112.03 & 1247.00 & $1893-1940$ & $\mathrm{D}$ \\
\hline & & 244055 & 24144 & 46.58 & 112.00 & 1187.00 & 1940-1992 & B \\
\hline & Kalispell & 244563 & 99999 & 48.20 & 114.30 & 906.00 & 1899-1949 & $\mathrm{D}$ \\
\hline & & 244558 & 24146 & 48.30 & 114.27 & 904.00 & 1949-1992 & A \\
\hline & Miles City & 245685 & 99999 & 46.40 & 105.82 & 719.00 & $1893-1968$ & $\mathrm{E}$ \\
\hline & & 245690 & 24037 & 46.43 & 105.87 & 802.00 & 1939-1990 & $\mathrm{C}$ \\
\hline & Missoula & 24MSOC & 94105 & 46.87 & 114.00 & 981.00 & $1935-1944$ & C \\
\hline & & 245745 & 24153 & 46.92 & 114.08 & 973.00 & 1939-1992 & B \\
\hline \multirow[t]{3}{*}{$\mathrm{NC}$} & Asheville & 310301 & 13872 & 35.60 & 82.53 & 683.00 & $1902-1964$ & $\mathrm{D}$ \\
\hline & & 310300 & 03812 & 35.43 & 82.55 & 652.00 & 1964-1992 & $A$ \\
\hline & Cape Hatteras & 311458 & 93729 & 35.27 & 75.55 & 3.00 & $1957-1992$ & A \\
\hline
\end{tabular}


Table 1 (continued)

\begin{tabular}{|c|c|c|c|c|c|c|c|c|}
\hline State $^{a}$ & $\begin{array}{l}\text { Station } \\
\text { name }\end{array}$ & $\begin{array}{c}\text { NCDC }^{b} \\
\text { station no. }\end{array}$ & $\begin{array}{l}\text { WBAN } \\
\text { sta. no. }\end{array}$ & $\begin{array}{l}\text { Lat. }^{d} \\
\left({ }^{\circ} \mathrm{N}\right)\end{array}$ & $\begin{array}{l}\text { Long. }{ }^{d} \\
\left({ }^{\circ} \mathrm{W}\right)\end{array}$ & $\begin{array}{l}\text { Elev. }^{e} \\
(\mathrm{~m})\end{array}$ & $\begin{array}{l}\text { Period } \\
\text { of Record }\end{array}$ & $\begin{array}{c}\text { Record } \\
\text { Completeness }\end{array}$ \\
\hline \multirow[t]{13}{*}{ NC } & \multirow[t]{2}{*}{ Charlotte } & 311695 & 93849 & 35.23 & 80.85 & 226.00 & $1893-1951$ & $\mathrm{D}$ \\
\hline & & 311690 & 13881 & 35.22 & 80.93 & 213.00 & 1940-1992 & $\mathrm{E}$ \\
\hline & \multirow[t]{2}{*}{ Greensboro } & 31GSOV & 99999 & 36.07 & 79.82 & 257.00 & 1929-1946 & $\mathrm{D}$ \\
\hline & & 313630 & 13723 & 36.08 & 79.95 & 270.00 & $1942-1992$ & B \\
\hline & Hatteras & 313897 & 13745 & 35.22 & 75.68 & 1.00 & 1893-1988 & $\mathrm{D}$ \\
\hline & Raleigh/Durham & 31RDUA & 99999 & 35.78 & 78.63 & 122.00 & 1941-1944 & $\mathrm{D}$ \\
\hline & \multirow{2}{*}{ Raleigh } & 317079 & 13784 & 35.45 & 78.62 & 105.00 & $1893-1954$ & $\mathrm{E}$ \\
\hline & & 317069 & 13722 & 35.87 & 78.78 & 115.00 & 1944-1992 & B \\
\hline & \multirow[t]{5}{*}{ Wilmington } & 319462 & 99999 & 34.23 & 77.95 & 9.75 & $1894-1931$ & $\mathrm{D}$ \\
\hline & & 319462 & 99999 & 34.23 & 77.95 & 2.44 & $1931-1951$ & $\mathrm{C}$ \\
\hline & & 319457 & 13748 & 34.23 & 77.95 & 9.14 & $1951-1963$ & A \\
\hline & & 319457 & 13748 & 34.23 & 77.95 & 8.53 & 1963-1979 & A \\
\hline & & 319457 & 13748 & 34.23 & 77.95 & 9.14 & 1979-1992 & A \\
\hline \multirow[t]{7}{*}{ ND } & \multirow[t]{2}{*}{ Bismarck } & 320814 & 99999 & 46.80 & 100.80 & 509.00 & 1893-1953 & $\mathrm{E}$ \\
\hline & & 320819 & 24011 & 46.77 & 100.75 & 502.00 & 1939-1992 & B \\
\hline & \multirow[t]{2}{*}{ Devils Lake } & 322158 & 14912 & 48.12 & 98.87 & 448.00 & 1904-1948 & C \\
\hline & & 322158 & 14912 & 48.12 & 98.87 & 446.00 & $1948-1963$ & $\mathrm{E}$ \\
\hline & Fargo & 322859 & 14914 & 46.90 & 96.80 & 274.00 & 1938-1992 & $\mathrm{A}$ \\
\hline & \multirow[t]{2}{*}{ Williston } & $32 I S N C$ & 24014 & 48.15 & 103.62 & 572.00 & 1893-1962 & $\mathrm{C}$ \\
\hline & & 329425 & 94014 & 48.18 & 103.63 & 579.00 & $1962-1992$ & A \\
\hline \multirow[t]{10}{*}{ NE } & \multirow{3}{*}{$\begin{array}{l}\text { Grand Island } \\
\text { Lincoln }\end{array}$} & 253395 & 14935 & 40.97 & 98.30 & 561.00 & $1948-1992$ & A \\
\hline & & 254815 & 14971 & 40.82 & 96.70 & 351.00 & $1897-1992$ & $\mathrm{E}$ \\
\hline & & 254795 & 14939 & 40.85 & 96.75 & 363.00 & 1939-1992 & $\mathrm{E}$ \\
\hline & \multirow[t]{2}{*}{ North Platte } & 256070 & 24055 & 41.13 & 100.75 & 855.00 & 1893-1949 & $\mathrm{D}$ \\
\hline & & 256065 & 24023 & 41.13 & 100.68 & 846.00 & 1948-1992 & A \\
\hline & \multirow[t]{3}{*}{ Omaha } & 250MAV & 14983 & 41.27 & 95.93 & 311.00 & 1893-1935 & $\mathrm{C}$ \\
\hline & & 256255 & 14942 & 41.30 & 95.90 & 299.00 & $1935-1992$ & C \\
\hline & & 256260 & 94918 & 41.37 & 96.02 & 399.00 & 1963-1992 & $\mathrm{C}$ \\
\hline & \multirow[t]{2}{*}{ Valentine } & $25 \mathrm{VINC}$ & 24032 & 42.88 & 100.55 & 787.00 & $1893-1955^{8}$ & $\mathrm{E}$ \\
\hline & & 258760 & 24032 & 42.87 & 100.55 & 789.00 & $1963-1992^{8}$ & A \\
\hline \multirow[t]{3}{*}{$\mathrm{NH}$} & \multirow[t]{2}{*}{ Concord } & $27 \mathrm{CONC}$ & 94756 & 43.20 & 71.53 & 82.00 & $1902-1941$ & $D$ \\
\hline & & 271683 & 14745 & 43.20 & 71.50 & 105.00 & 1941-1992 & A \\
\hline & Mt. Washington & 275639 & 14755 & 44.27 & 71.30 & 1909.00 & $1937-1992$ & B \\
\hline \multirow[t]{5}{*}{ NJ } & \multirow[t]{2}{*}{ Atlantic City } & 280325 & 13724 & 39.38 & 74.43 & 3.00 & 1893-1992 & $E$ \\
\hline & & 280311 & 93730 & 39.45 & 74.57 & 42.00 & $1958-1992$ & A \\
\hline & \multirow[t]{3}{*}{ Trenton } & 288883 & 14773 & 40.22 & 74.77 & 58.00 & 1913-1932 & $D$ \\
\hline & & 288883 & 14773 & 40.22 & 74.77 & 42.00 & $1933-1948$ & $\mathrm{D}$ \\
\hline & & 288883 & 14773 & 40.22 & 74.77 & 57.00 & $1948-1981$ & $E$ \\
\hline
\end{tabular}


Table 1 (continued)

\begin{tabular}{|c|c|c|c|c|c|c|c|c|}
\hline State $^{\alpha}$ & $\begin{array}{l}\text { Station } \\
\text { name }\end{array}$ & $\begin{array}{c}\mathrm{NCDC}^{b} \\
\text { station no. }\end{array}$ & $\begin{array}{l}\text { WBAN } \\
\text { sta. no. }\end{array}$ & $\begin{array}{l}\text { Lat. }^{d} \\
\left({ }^{\circ} \mathrm{N}\right)\end{array}$ & $\begin{array}{l}\text { Long. }{ }^{d} \\
\left({ }^{\circ} \mathrm{W}\right)\end{array}$ & $\begin{array}{l}\text { Elev. } \\
(\mathrm{m})\end{array}$ & $\begin{array}{c}\text { Period } \\
\text { of Record }\end{array}$ & $\begin{array}{c}\text { Record } \\
\text { Completeness }\end{array}$ \\
\hline \multirow[t]{5}{*}{ NM } & Albuquerque & $29 A B Q C$ & 23073 & 35.08 & 106.65 & 1512.00 & 1919-1932 & D \\
\hline & & 290234 & 23050 & 35.05 & 106.62 & 1619.00 & 1933-1992 & $\mathrm{C}$ \\
\hline & Roswell & 29ROWC & 99999 & 33.40 & 104.53 & 1086.00 & 1904-1947 & $\mathrm{D}$ \\
\hline & & 297609 & 23043 & 33.40 & 104.53 & 1101.00 & $1947-1972$ & B \\
\hline & & 297610 & 23009 & 33.30 & 104.53 & 1119.00 & 1973-1992 & A \\
\hline \multirow[t]{7}{*}{ NV } & Ely & 262631 & 23154 & 39.28 & 114.85 & 1909.00 & 1938-1992 & $\mathrm{C}$ \\
\hline & Las Vegas & 264434 & 23173 & 36.23 & 115.03 & 573.00 & 1939-1948 & B \\
\hline & & 264436 & 23169 & 36.08 & 115.17 & 659.00 & 1949-1992 & A \\
\hline & Reno & 26RNOC & 99999 & 39.53 & 119.82 & 1369.00 & $1905-1942$ & D \\
\hline & & 266779 & 23185 & 39.50 & 119.78 & 1343.00 & 1939-1992 & B \\
\hline & Winnemucca & 26WMCC & 24129 & 40.97 & 117.72 & 1307.00 & 1893-1949 & $\mathrm{D}$ \\
\hline & 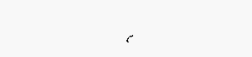 & 269171 & 24128 & 40.90 & 117.80 & 1310.00 & 1949-1992 & A \\
\hline \multirow[t]{14}{*}{ NY } & Albany & 300047 & 14796 & 42.65 & 73.75 & 6.00 & 1893-1942 & $\mathrm{C}$ \\
\hline & & 300042 & 14735 & 42.75 & 73.80 & 84.00 & 1938-1992 & B \\
\hline & Binghamton & 300691 & 14798 & 42.10 & 75.92 & 262.00 & $1896-1968$ & $\mathrm{E}$ \\
\hline & & 300686 & 14738 & 42.08 & 76.10 & 252.00 & $1942-1951$ & $\mathrm{C}$ \\
\hline & & 300687 & 04725 & 42.22 & 75.98 & 488.00 & 1951-1992 & B \\
\hline & Buffalo & 30BUFC & 94753 & 42.88 & 78.88 & 184.00 & $1893-1943$ & B \\
\hline & & 301012 & 14733 & 42.93 & 78.73 & 215.00 & 1943-1992 & B \\
\hline & NYC & 305816 & 94706 & 40.77 & 73.98 & 21.00 & $1893-1948$ & $\mathrm{C}$ \\
\hline & & 305811 & 14732 & 40.77 & 73.90 & 3.00 & 1947-1992 & $\mathrm{A}$ \\
\hline & Oswego & 306314 & 14759 & 43.45 & 76.52 & 102.00 & $1893-1948$ & D \\
\hline & Rochester & 30ROCC & 94777 & 43.15 & 77.62 & 151.00 & $1893-1940$ & $\mathrm{C}$ \\
\hline & & 307167 & 14768 & 43.12 & 77.67 & 167.00 & $1940-1992$ & B \\
\hline & Syracuse & 30SYRC & 94781 & 43.05 & 76.15 & 122.00 & $1902-1940$ & $\mathrm{C}$ \\
\hline & & 308383 & 14771 & 43.12 & 76.12 & 128.00 & $1940-1992$ & B \\
\hline \multirow[t]{12}{*}{$\mathrm{OH}$} & Akron & 330063 & 14813 & 41.03 & 81.45 & 318.00 & 1944-1951 & $\mathrm{D}$ \\
\hline & Akron/Canton & 330058 & 14895 & 40.92 & 81.43 & 368.00 & $1948-1992$ & A \\
\hline & Cincinnati & 331581 & 03871 & 39.10 & 84.52 & 169.00 & $1893-1970$ & $E$ \\
\hline & & 331561 & 93890 & 39.15 & 84.52 & 232.00 & $1915-1980$ & $\mathrm{E}$ \\
\hline & Cleveland & 331662 & 14882 & 41.50 & 81.70 & 198.00 & $1893-1941$ & $\mathrm{C}$ \\
\hline & & 331657 & 14820 & 41.42 & 81.87 & 237.00 & $1941-1992$ & B \\
\hline & Columbus & 331788 & 93891 & 39.97 & 83.00 & 221.00 & 1893-1973 & $\mathrm{D}$ \\
\hline & & 331786 & 14821 & 40.00 & 82.88 & 247.00 & 1939-1992 & $\mathrm{E}$ \\
\hline & Dayton & 33DAYC & 99999 & 39.77 & 84.20 & 226.00 & $1911-1943$ & $\mathrm{C}$ \\
\hline & & 338598 & 99999 & 39.90 & 84.20 & 305.00 & $1942-1950$ & $\mathrm{C}$ \\
\hline & & 332075 & 93815 & 39.90 & 84.20 & 306.00 & $1950-1992$ & A \\
\hline & Sandusky & 337447 & 14846 & 41.45 & 82.72 & 192.00 & $1893-1963$ & $\mathrm{E}$ \\
\hline
\end{tabular}


Table 1 (continued)

\begin{tabular}{|c|c|c|c|c|c|c|c|c|}
\hline State $^{a}$ & $\begin{array}{l}\text { Station } \\
\text { name }\end{array}$ & $\begin{array}{c}\mathrm{NCDC}^{b} \\
\text { station no. }\end{array}$ & $\begin{array}{l}\text { WBAN }^{c} \\
\text { sta. no. }\end{array}$ & $\begin{array}{l}\text { Lat. }^{d} \\
\left({ }^{\circ} \mathrm{N}\right)\end{array}$ & $\begin{array}{l}\text { Long. }{ }^{d} \\
\left({ }^{\circ} \mathrm{W}\right)\end{array}$ & $\begin{array}{l}\text { Elev. }^{e} \\
(\mathrm{~m})\end{array}$ & $\begin{array}{l}\text { Period } \\
\text { of Record }\end{array}$ & $\begin{array}{c}\text { Record } \\
\text { Completeness }\end{array}$ \\
\hline \multirow[t]{3}{*}{$\mathrm{OH}$} & Toledo & 338366 & 14889 & 41.65 & 83.53 & 180.00 & $1893-1943$ & $\mathrm{C}$ \\
\hline & & 338356 & 14849 & 41.57 & 83.47 & 190.00 & 1939-1954 & B \\
\hline & & 338357 & 94830 & 41.58 & 83.80 & 204.00 & 1955-1992 & A \\
\hline \multirow[t]{3}{*}{ OK } & Oklahoma City & 346656 & 93954 & 35.48 & 97.53 & 382.00 & $1893-1948$ & A \\
\hline & & 346661 & 13967 & 35.40 & 97.60 & 390.00 & $1948-1992$ & A \\
\hline & Tulsa & 348992 & 13968 & 36.18 & 95.90 & 204.00 & $1938-1992$ & $E$ \\
\hline \multirow[t]{9}{*}{ OR } & Baker & 350417 & 24199 & 44.77 & 117.85 & 1057.00 & $1893-1939$ & $E$ \\
\hline & & 350412 & 24130 & 44.83 & 117.82 & 1028.00 & $1939-1948$ & $\mathrm{D}$ \\
\hline & & 350412 & 24130 & 44.83 & 117.82 & 1026.00 & $1948-1953$ & $\mathrm{C}$ \\
\hline & Medford & 35MFRC & 99999 & 42.33 & 122.85 & 420.00 & 1929 & $G$ \\
\hline & & 355429 & 24225 & 42.38 & 122.88 & 396.00 & $1930-1992$ & $\mathrm{C}$ \\
\hline & Portland & 356761 & 27274 & 45.53 & 122.67 & 9.00 & $1893-1973$ & $\mathrm{D}$ \\
\hline & & 356751 & 24229 & 45.60 & 122.60 & 6.00 & $1940-1992$ & B \\
\hline & Roseberg & 357326 & 24231 & 43.23 & 123.37 & 155.00 & $1893-1952$ & $\mathrm{E}$ \\
\hline & & 357326 & 24231 & 43.23 & 123.37 & 154.00 & $1953-1964$ & A \\
\hline \multirow[t]{14}{*}{ PA } & Erie & 362677 & 14893 & 42.12 & 80.08 & 200.00 & 1893-1952 & $\mathrm{D}$ \\
\hline & & 362682 & 14860 & 42.08 & 80.18 & 223.00 & 1946-1992 & $\mathrm{E}$ \\
\hline & Harrisburg & 363710 & 94718 & 40.27 & 76.88 & 102.00 & $1893-1942$ & D \\
\hline & & 363699 & 14751 & 40.22 & 76.85 & 104.00 & 1939-1991 & $\mathrm{C}$ \\
\hline & Philadelphia & 366909 & 13779 & 39.95 & 75.15 & 11.00 & $1893-1964$ & $\mathrm{E}$ \\
\hline & & 366889 & 13739 & 39.88 & 75.23 & 3.00 & $1940-1992$ & $\mathrm{C}$ \\
\hline & Pittsburgh & 366997 & 14861 & 40.45 & 80.00 & 228.00 & $1893-1978$ & $\mathrm{E}$ \\
\hline & & 366992 & 14762 & 40.35 & 79.93 & 382.00 & 1939-1952 & B \\
\hline & & 366993 & 94823 & 40.50 & 80.22 & 351.00 & $1952-1992$ & $\mathrm{~B}$ \\
\hline & Reading & 367318 & 14767 & 40.33 & 75.97 & 98.00 & $1912-1932$ & $\mathrm{D}$ \\
\hline & & 367318 & 14767 & 40.33 & 75.97 & 157.00 & $1933-1939$ & $\mathrm{D}$ \\
\hline & & 367318 & 14767 & 40.33 & 75.97 & 88.00 & $1940-1969$ & $\mathrm{E}$ \\
\hline & Scranton & 367902 & 14769 & 41.42 & 75.67 & 227.00 & $1900-1954$ & $E$ \\
\hline & Wilkes-Barre & 367905 & 14777 & 41.33 & 75.73 & 283.00 & $1955-1992$ & A \\
\hline \multirow[t]{4}{*}{ RI } & Block Island & 37BIDC & 14799 & 41.17 & 71.57 & 11.00 & $1893-1950^{g}$ & $\mathrm{D}$ \\
\hline & & 370896 & 94793 & 41.17 & 71.58 & 34.00 & $1962-1992^{8}$ & $\mathrm{E}$ \\
\hline & Providence & 376703 & 94707 & 41.83 & 71.42 & 4.00 & $1904-1953$ & $\mathrm{D}$ \\
\hline & & 376698 & 14765 & 41.73 & 71.43 & 16.00 & 1942-1992 & $\mathrm{E}$ \\
\hline \multirow[t]{4}{*}{ SC } & Charleston & 381549 & 13782 & 32.78 & 79.93 & 3.00 & 1893-1992 & $\mathrm{E}$ \\
\hline & & 381544 & 13880 & 32.9 & 80.03 & 13.00 & $1948-1992$ & $A$ \\
\hline & Columbia & 381944 & 93851 & 34.00 & 81.05 & 101.00 & $1899-1954$ & $\mathrm{D}$ \\
\hline & & 381939 & 13883 & 33.95 & 81.12 & 65.00 & $1948-1992$ & A \\
\hline
\end{tabular}


Table 1 (continued)

\begin{tabular}{|c|c|c|c|c|c|c|c|c|}
\hline State $^{a}$ & $\begin{array}{l}\text { Station } \\
\text { name }\end{array}$ & $\begin{array}{c}\mathrm{NCDC}^{b} \\
\text { station no. }\end{array}$ & $\begin{array}{l}\text { WBAN }^{c} \\
\text { sta. no. }\end{array}$ & $\begin{array}{l}\text { Lat. }^{d} \\
\left({ }^{\circ} \mathrm{N}\right)\end{array}$ & $\begin{array}{l}\text { Long. }{ }^{d} \\
\left({ }^{\circ} \mathrm{W}\right)\end{array}$ & $\begin{array}{l}\text { Elev. }{ }^{e} \\
\text { (m) }\end{array}$ & $\begin{array}{l}\text { Period } \\
\text { of Record }\end{array}$ & $\begin{array}{c}\text { Record } \\
\text { Completeness }\end{array}$ \\
\hline \multirow[t]{3}{*}{ SC } & $\begin{array}{l}\text { Greenville/ } \\
\text { Spartanburg }\end{array}$ & 383747 & 03870 & 34.90 & 82.22 & 297.00 & 1962-1992 & B \\
\hline & Greenville & 383742 & 13886 & 34.85 & 82.35 & 310.00 & $1942-1962$ & B \\
\hline & Greer & $38 \mathrm{GSPC}$ & 99999 & 34.83 & 82.40 & 296.00 & $1917-1941$ & $\mathrm{C}$ \\
\hline \multirow[t]{4}{*}{$\mathrm{SD}$} & Huron & $39 \mathrm{HONC}$ & 14968 & 44.37 & 98.22 & 389.00 & $1893-1941$ & $\mathrm{C}$ \\
\hline & & 394127 & 14936 & 44.38 & 98.22 & 391.00 & $1933-1992$ & A \\
\hline & Rapid City & 396947 & 24056 & 44.07 & 103.20 & 988.00 & 1906-1942 & $\mathrm{C}$ \\
\hline & & 396937 & 24026 & 44.05 & 103.07 & 964.00 & 1939-1992 & B \\
\hline \multirow[t]{8}{*}{$\mathrm{TN}$} & Chattanooga & 40CHAC & 99999 & 35.07 & 85.23 & 225.00 & $1893-1940^{8}$ & D \\
\hline & & 401656 & 13882 & 35.03 & 85.20 & 211.00 & $1942-1992^{8}$ & B \\
\hline & Knoxville & 40TYSC & 99999 & 35.97 & 83.92 & 281.00 & 1893-1942 & $\mathrm{C}$ \\
\hline & & 404950 & 13891 & 35.80 & 84.00 & 289.00 & 1938-1992 & B \\
\hline & Memphis & 405964 & 93916 & 35.15 & 90.05 & 83.00 & $1893-1965$ & $\mathrm{D}$ \\
\hline & & 405954 & 13893 & 35.05 & 90.00 & 87.00 & 1940-1992 & $\mathrm{B}$ \\
\hline & Nashville & 406407 & 93858 & 36.17 & 86.78 & 152.00 & $1893-1950$ & $\mathrm{D}$ \\
\hline & & 406402 & 13897 & 36.12 & 86.68 & 177.00 & 1940-1992 & $\mathrm{C}$ \\
\hline \multirow[t]{21}{*}{$\mathrm{TX}$} & Abilene & 41ABIC & 93922 & 32.45 & 99.73 & 526.00 & 1893-1944 & $\mathrm{D}$ \\
\hline & & 410016 & 13962 & 32.42 & 99.68 & 544.00 & 1944-1992 & $\mathrm{B}$ \\
\hline & Amarillo & 41AMAC & 23075 & 35.22 & 101.83 & 1115.00 & $1893-1941$ & $\mathrm{D}$ \\
\hline & & 410211 & 23047 & 35.23 & 101.70 & 1095.00 & 1939-1992 & $\mathrm{C}$ \\
\hline & Austin & 41AUSC & 93923 & 30.28 & 97.73 & 151.00 & $1928-1942^{g}$ & $\mathrm{~B}$ \\
\hline & & 410428 & 13958 & 30.30 & 97.70 & 182.00 & $1944-1992^{8}$ & A \\
\hline & Big Spring & 410786 & 23041 & 32.23 & 101.50 & 773.00 & $1932-1949$ & $\mathrm{E}$ \\
\hline & & 410786 & 23041 & 32.23 & 101.50 & 784.00 & $1950-1953$ & B \\
\hline & Corpus Christi & 412014 & 12940 & 27.80 & 97.40 & 5.00 & 1895-1948 & $\mathrm{E}$ \\
\hline & & 412015 & 12924 & 27.77 & 97.50 & 13.00 & 1948-1992 & $\mathrm{A}$ \\
\hline & $\begin{array}{l}\text { Dallas- } \\
\text { Fort Worth }\end{array}$ & 412242 & 03927 & 32.90 & 97.03 & 168.00 & 1974-1992 & B \\
\hline & Dallas & 41DALC & 93928 & 32.77 & 96.78 & 132.00 & $1915-1940^{g}$ & $\mathrm{D}$ \\
\hline & & 412244 & 13960 & 32.85 & 96.85 & 145.00 & $1942-1992^{8}$ & $\mathrm{E}$ \\
\hline & Del Rio & 412357 & 22004 & 29.37 & 100.90 & 287.00 & 1909-1963 & $\mathrm{E}$ \\
\hline & & 412360 & 22010 & 29.37 & 100.92 & 314.00 & 1963-1992 & A \\
\hline & El Paso & 41EPLC & 23080 & 31.78 & 106.50 & 1131.00 & $1893-1942$ & $\mathrm{D}$ \\
\hline & & 412797 & 23044 & 31.80 & 106.40 & 1195.00 & 1943-1992 & $\mathrm{B}$ \\
\hline & Fort Worth & 413285 & 99999 & 32.75 & 97.33 & 188.00 & $1898-1939$ & $\mathrm{D}$ \\
\hline & & 413284 & 13961 & 32.82 & 97.35 & 210.00 & $1940-1992$ & $\mathrm{E}$ \\
\hline & & 413283 & 99999 & 32.83 & 97.05 & 164.00 & 1953-1974 & $\mathrm{A}$ \\
\hline & Galveston & 413430 & 12944 & 29.30 & 94.80 & 2.00 & 1895-1992 & $\mathrm{E}$ \\
\hline
\end{tabular}


Table 1 (continued)

\begin{tabular}{|c|c|c|c|c|c|c|c|c|}
\hline State $^{a}$ & $\begin{array}{l}\text { Station } \\
\text { name }\end{array}$ & $\begin{array}{c}\text { NCDC } \\
\text { station no. }\end{array}$ & $\begin{array}{l}\text { WBAN }^{c} \\
\text { sta. no. }\end{array}$ & $\begin{array}{l}\text { Lat. }^{d} \\
\left({ }^{\circ} \mathrm{N}\right)\end{array}$ & $\begin{array}{l}\text { Long. }{ }^{d} \\
\left({ }^{\circ} \mathrm{W}\right)\end{array}$ & $\begin{array}{l}\text { Elev. }^{e} \\
(\mathrm{~m})\end{array}$ & $\begin{array}{l}\text { Period } \\
\text { of Record }\end{array}$ & $\begin{array}{c}\text { Record } \\
\text { Completeness }^{f}\end{array}$ \\
\hline \multirow[t]{9}{*}{$\mathrm{TX}$} & \multirow[t]{3}{*}{ Houston } & 414305 & 12945 & 25.77 & 95.37 & 12.00 & 1909-1988 & $\mathrm{E}$ \\
\hline & & 414307 & 12918 & 29.65 & 95.28 & 15.00 & $1948-1969$ & B \\
\hline & & 414300 & 12960 & 29.97 & 95.35 & 29.00 & 1969-1992 & $\mathrm{A}$ \\
\hline & Lubbock & 415411 & 23042 & 33.65 & 101.82 & 992.00 & $1938-1992$ & $\mathrm{E}$ \\
\hline & Palestine & 416757 & 93914 & 31.75 & 95.67 & 158.00 & 1893-1952 & $\mathrm{E}$ \\
\hline & \multirow[t]{2}{*}{ Port Arthur } & 417173 & 12943 & 29.87 & 93.93 & 1.52 & $1918-1953$ & $\mathrm{C}$ \\
\hline & & 417174 & 12917 & 29.95 & 94.02 & 4.00 & $1948-1992$ & A \\
\hline & \multirow[t]{2}{*}{ San Antonio } & 41SATC & 12938 & 29.45 & 98.47 & 199.00 & $1895-1940$ & $\mathrm{C}$ \\
\hline & & 417945 & 12921 & 29.53 & 98.47 & 242.00 & $1940-1992$ & B \\
\hline \multirow[t]{3}{*}{ UT } & Milford & 425654 & 23176 & 38.43 & 113.02 & 1533.00 & $1938-1992$ & $D$ \\
\hline & \multirow[t]{2}{*}{ Salt Lake City } & 427603 & 24175 & 40.77 & 111.90 & 1298.00 & 1893-1954 & $\mathrm{E}$ \\
\hline & & 427598 & 24127 & 40.78 & 111.95 & 1287.00 & 1935-1992 & $\mathrm{C}$ \\
\hline \multirow[t]{10}{*}{ VA } & \multirow[t]{2}{*}{ Cape Henry } & 441362 & 13727 & 36.93 & 76.00 & 2.00 & 1899-1901 & $\mathrm{D}$ \\
\hline & & 441362 & 13727 & 36.93 & 76.00 & 13.00 & $1902-1948$ & $\mathrm{E}$ \\
\hline & \multirow[t]{2}{*}{ Washington, DC } & 448904 & 93725 & 38.90 & 77.05 & 22.00 & 1893-1951 & $\mathrm{D}$ \\
\hline & & 448906 & 13743 & 38.85 & 77.03 & 3.00 & 1945-1992 & B \\
\hline & \multirow[t]{2}{*}{ Lynchburg } & 44LYHC & 93708 & 37.42 & 79.15 & 187.00 & 1893-1944 & D \\
\hline & & 445120 & 13733 & 37.33 & 79.20 & 280.00 & 1944-1992 & B \\
\hline & \multirow[t]{2}{*}{ Norfolk } & 446144 & 13778 & 36.87 & 76.28 & 3.00 & $1893-1965$ & $\mathrm{D}$ \\
\hline & & 446139 & 13737 & 36.90 & 76.20 & 7.00 & 1941-1992 & B \\
\hline & \multirow[t]{2}{*}{ Richmond } & 447206 & 13780 & 37.53 & 77.42 & 49.00 & $1898-1953$ & D \\
\hline & & 447201 & 13740 & 37.50 & 77.33 & 50.00 & $1925-1992$ & B \\
\hline \multirow[t]{2}{*}{ VT } & \multirow[t]{2}{*}{ Burlington } & 43BTVC & 94712 & 44.48 & 73.18 & 121.00 & $1906-1943$ & $\mathrm{C}$ \\
\hline & & 431081 & 14742 & 44.47 & 73.15 & 101.00 & 1943-1992 & B \\
\hline \multirow[t]{11}{*}{ WA } & North Head & 455932 & 24226 & 46.30 & 124.08 & 64.00 & $1903-1953$ & $\mathrm{E}$ \\
\hline & Seattle/Tacoma & 457473 & 24233 & 47.45 & 122.30 & 137.00 & 1944-1992 & B \\
\hline & \multirow[t]{2}{*}{ Seattle } & 457488 & 99999 & 47.60 & 122.33 & 4.00 & 1894-1972 & $\mathrm{D}$ \\
\hline & & 457458 & 24281 & 47.65 & 122.30 & 6.00 & 1973-1992 & A \\
\hline & \multirow[t]{2}{*}{ Spokane } & 45SKAC & 99999 & 47.67 & 117.42 & 578.00 & $1893-1940$ & $\mathrm{C}$ \\
\hline & & 457938 & 24157 & 47.63 & 117.53 & 718.00 & 1939-1992 & $\mathrm{C}$ \\
\hline & \multirow[t]{3}{*}{ Tacoma } & 458286 & 24239 & 47.25 & 122.43 & 65.00 & $1897-1922$ & $\mathrm{D}$ \\
\hline & & 458286 & 24239 & 47.25 & 122.43 & 77.00 & $1923-1948$ & $\mathrm{E}$ \\
\hline & & 458286 & 24239 & 47.25 & 122.43 & 82.00 & $1948-1953$ & $\mathrm{E}$ \\
\hline & Tatoosh Island & 458332 & 24240 & 48.38 & 124.73 & 35.00 & $1893-1966$ & $E$ \\
\hline & Walla Walla & 458931 & 94103 & 46.03 & 118.33 & 289.00 & 1893-1988 & $\mathrm{E}$ \\
\hline \multirow[t]{2}{*}{ WI } & \multirow[t]{2}{*}{ Green Bay } & 47GRBC & 14831 & 44.52 & 87.98 & 181.00 & 1893-1949 & $\mathrm{C}$ \\
\hline & & 473269 & 14898 & 44.48 & 88.13 & 208.00 & 1949-1992 & A \\
\hline
\end{tabular}


Table 1 (continued)

\begin{tabular}{|c|c|c|c|c|c|c|c|c|}
\hline State $^{a}$ & $\begin{array}{l}\text { Station } \\
\text { name }\end{array}$ & $\begin{array}{c}\mathrm{NCDC}^{b} \\
\text { station no. }\end{array}$ & $\begin{array}{l}\text { WBAN }^{c} \\
\text { sta. no. }\end{array}$ & $\begin{array}{l}\text { Lat. }^{d} \\
\left({ }^{\circ} \mathrm{N}\right)\end{array}$ & $\begin{array}{l}\text { Long. }{ }^{d} \\
\left({ }^{\circ} \mathrm{W}\right)\end{array}$ & $\begin{array}{l}\text { Elev. }{ }^{e} \\
(\mathrm{~m})\end{array}$ & $\begin{array}{l}\text { Period } \\
\text { of Record }\end{array}$ & $\begin{array}{c}\text { Record } \\
\text { Completeness }\end{array}$ \\
\hline \multirow[t]{6}{*}{ WI } & \multirow[t]{2}{*}{ La Crosse } & 474375 & 14960 & 43.82 & 91.25 & 205.00 & $1893-1951$ & D \\
\hline & & 474370 & 14920 & 43.87 & 91.25 & 200.00 & 1939-1992 & $\mathrm{B}$ \\
\hline & \multirow[t]{2}{*}{ Madison } & 474966 & 14887 & 43.08 & 89.40 & 286.00 & $1904-1963$ & $E$ \\
\hline & & 474961 & 14837 & 43.13 & 89.33 & 262.00 & 1939-1992 & B \\
\hline & \multirow[t]{2}{*}{ Milwaukee } & 475484 & 14888 & 43.03 & 87.90 & 189.00 & $1893-1953$ & $\mathrm{D}$ \\
\hline & & 475479 & 14839 & 42.95 & 87.90 & 205.00 & 1939-1992 & B \\
\hline \multirow[t]{5}{*}{ WV } & \multirow[t]{2}{*}{ Elkins } & 46EKNC & 99999 & 38.93 & 79.85 & 587.00 & $1899-1944$ & $\mathrm{C}$ \\
\hline & & 462718 & 13729 & 38.87 & 79.85 & 600.00 & 1944-1992 & $\mathrm{E}$ \\
\hline & \multirow[t]{3}{*}{ Parkersburg } & 466859 & 13867 & 39.27 & 81.57 & 187.00 & 1893-1919 & $\mathrm{D}$ \\
\hline & & 466859 & 13867 & 39.27 & 81.57 & 205.00 & $1919-1948$ & $\mathrm{D}$ \\
\hline & & 466859 & 13867 & 39.27 & 81.57 & 201.00 & $1948-1982$ & $\mathrm{E}$ \\
\hline \multirow[t]{6}{*}{ WY } & \multirow[t]{2}{*}{ Cheyenne } & 48CYSC & 24068 & 41.13 & 104.80 & 1852.00 & 1893-1935 & B \\
\hline & & 481675 & 24018 & 41.15 & 104.82 & 1866.00 & $1935-1992$ & $\mathrm{~B}$ \\
\hline & \multirow[t]{2}{*}{ Lander } & 48LNDC & 24075 & 42.83 & 108.73 & 1631.00 & $1893-1946$ & $\mathrm{C}$ \\
\hline & & 485390 & 24021 & 42.82 & 108.73 & 1637.00 & 1946-1992 & B \\
\hline & \multirow[t]{2}{*}{ Sheridan } & 48SHRC & 24058 & 44.80 & 106.95 & 1150.00 & $1907-1940$ & B \\
\hline & & 488155 & 24029 & 44.70 & 7106.97 & 1209.00 & $1940-1992$ & B \\
\hline
\end{tabular}

${ }^{a}$ See Appendix A for a list of the state codes, state abbreviations, and state names.

National Climatic Data Center.

"Weather Bureau Army Navy station number. "99999" indicates no WBAN number assigned.

"Values for latitude and longitude are in decimal degrees. The value " $-99.99 "$ indicates a missing coordinate.

Values for elevation are in meters above sea level. The value " -99.99 " indicates a missing elevation.

The Record Completeness categories grossly characterize the usefulness of the data as follows:

$\mathrm{A}=$ There are seven or more months of data in every year of the particular station number's record;

$\mathrm{B}=$ The first year or two of the record is missing one or two months of data, or one or two years in the middle or at the end of the record is missing one month of data, but the remaining years in the station record have seven or more months of data;

$\mathrm{C}=$ Several years are missing one to three months of data;

$\mathrm{D}=$ Many years are missing at least one and usually many months of data;

$\mathrm{E}=$ One or more years are missing in the station record;

$\mathrm{F}=$ The one-year station record has seven months of winter season data; and

$\mathrm{G}=$ The one-year station record has less than seven months of winter season data.

${ }^{8}$ Station move(s) resulted in the historical record for this general location being discontinuous. 


\section{QUALITY ASSURANCE OF THE U.S. DAILY SNOW DEPTH DATABASE}

An important part of the numeric data packaging process at the Carbon Dioxide Information Analysis Center (CDIAC) involves the quality assurance (QA) of data before distribution. Data received by CDIAC are rarely in a condition that would permit immediate distribution, regardless of their source. To guarantee data of the highest possible quality, CDIAC performs extensive QA reviews that involve examining the data for completeness, reasonableness, and accuracy. NCDC conducted extensive manual and automated QA assessments of the snow depth data before sending the data to CDIAC. Although the data sent by NCDC were in excellent condition, CDIAC still conducted QA checks on the data and found a few minor discrepancies. The following summarizes the QA work performed by NCDC and CDIAC, respectively.

\section{NCDC QA CHECKS AND ADJUSTMENTS}

1. NCDC performed a manual review of

- snow depths that were 40 or more in. greater than the previous day's depth and

- snow depths from months whose total increase in snow depth (plus an additional $10 \mathrm{in}$.) over the previous month's snow depth was less than the reported snowfall total for the month.

2. Differences between daily snow depth values were computed and summed. This represented a quasi-snowfall total for the month, which was compared to an independent data set containing actual total monthly snowfall. Whenever the computed snowfall (estimated from snow depth differences) exceeded the actual total, a manual review was performed. Instances of "carryover" of snow depth between months were taken into account.

3. During the manual review process by NCDC, numerous instances were detected where the suspicious snow depth amounts were determined to include either drifted snow or sleet. In some cases, the editor could substitute an estimate which excluded the extra amount; this estimated value was assigned a data measurement flag of "E." If it was not possible to substitute an estimated value, the snow depth value was flagged with an " $L$ " to reflect the inclusion of sleet. No uniform method of detecting drifts or sleet was used, as additional parameters (e.g., temperature and present weather) would have been needed to conduct a comprehensive determination.

\section{CDIAC QA CHECKS AND MODIFICATIONS}

1. The logical record length of the snow depth data files was decreased from 402 to 270 characters. This was accomplished mainly by compressing the width of each daily data field so that it contained fewer blank characters.

2. Because each record in the snow depth database contains 31 daily data elements (to allow for 31 days in a month), elements pertaining to nonexistent dates were checked for missing data indicators with blank flag spaces (the prescribed conventions).

3. A few data measurement and data quality flags were found that were not included in the documentation provided by NCDC. Close examination of the records in question revealed 
that these undocumented flag values were likely the result of data entry errors. The records containing these flags were corrected and the information forwarded to NCDC for corroboration.

4. All data records were checked to ensure that the number of days in the month (specified in each record) was correct for the year and month of each record.

5. Records of stations with data for only seven months per year were examined to confirm that the seven months were the winter months. If not, the records were flagged with the appropriate record completeness flag (see Table 1).

6. Snow depths of 20 or more in. greater than the previous day's depth were examined for reasonableness, usually by checking the daily values for several days in sequence. In addition, values were often compared with daily snow depths from stations at nearby locations (where possible) for the period in question to look for a similar pattern in daily snow depths.

7. Snow depths of greater than $100 \mathrm{in}$. were checked to make sure these values were possible for the particular station location and date and were consistent with depths reported on earlier and later days for that station.

8. Questionable records, such as the few records with possible input errors, were compared with records in NCDC's Summary of the Day-First Order online database (NCDC Tape Deck 3210) (http://www.ncdc.noaa.gov/pub/data/fsod/).

9. In general, it was noted that most snow depth records from 1948 to the present have at least the seven winter months of data per year. 


\section{HOW TO OBTAIN THE DATA PACKAGE}

The U.S. daily snow depth database is available free of charge from CDIAC. The files are available on 8-mm tape or quarter-inch tape cartridge, via CDIAC's anonymous file transfer protocol (FTP) service, or via the World Wide Web. (Users are asked to contact CDIAC for assistance if these mediums are not suited to their particular computing environment.) A sampling of commands used to obtain the station inventory file (INVENT.DAT) from this database via FTP follows. CDIAC FTP server responses are shown in italics; user entries are bolded. For a full description of commands, contact CDIAC.

$>$ ftp cdiac.esd.ornl.gov or $>$ ftp 128.219.24.39

Name: anonymous

Password: YOU@your internet address

Guest login ok, access restrictions apply.

ftp> cd /pub/ndp059

$f t p>$ dir

$f t p>$ get invent.dat

ftp> quit

$f t p>$ Goodbye

The database can also be obtained via the World Wide Web. The uniform resource locator (URL) for CDIAC's homepage is http://cdiac.esd.ornl.gov.

For non-Internet data acquisitions, users may request data from CDIAC using the following information:

Address: $\quad$ Carbon Dioxide Information Analysis Center

Oak Ridge National Laboratory

Post Office Box 2008

Oak Ridge, Tennessee 37831-6335, U.S.A.

Telephone: $\quad 423-574-3645$ (Voice)

423-574-2232 (Fax)

Electronic Mail: Internet: cdiac@ornl.gov 


\section{REFERENCES}

Groisman, P. Y., and D. R. Easterling. 1994. Variability and trends of total precipitation and snowfall over the United States and Canada. J. Climate 7:184-205.

Intergovernmental Panel on Climate Change (IPCC). 1996. Climate Change 1995: The Science of Climate Change. J. T. Houghton, L. G. Meira Filho, B. A. Callander, N. Harris, A. Kattenberg, and K. Maskell (eds.). Cambridge University Press, Cambridge, U.K.

Karl, T. R., P. Y. Groisman, R. W. Knight, and R. R. Heim, Jr. 1993. Recent variations of snow cover and snowfall in North America and their relation to precipitation and temperature variations. J. Climate 6:1327-44.

Leathers, D. J., and D. A. Robinson. 1993. The association between extremes in North American snow cover extent and United States temperatures. J. Climate 6:1345-55.

Nicholls, N., G. V. Gruza, J. Jouzel, T. R. Karl, L. A. Ogallo, and D. E. Parker. 1996. Observed climate variability and change. pp. 133-92. In J. T. Houghton, L. G. Meira Filho, B. A. Callander, N. Harris, A. Kattenberg, and K. Maskell (eds.), IPCC, Climate Change 1995: The Science of Climate Change. Cambridge University Press, Cambridge, U.K.

Steurer, P. M., and T. R. Karl. 1991. Historical sunshine and cloud data in the United States. ORNL/CDIAC-43, NDP-021/R1. Carbon Dioxide Information Analysis Center, Oak Ridge National Laboratory, Oak Ridge, Tennessee. 


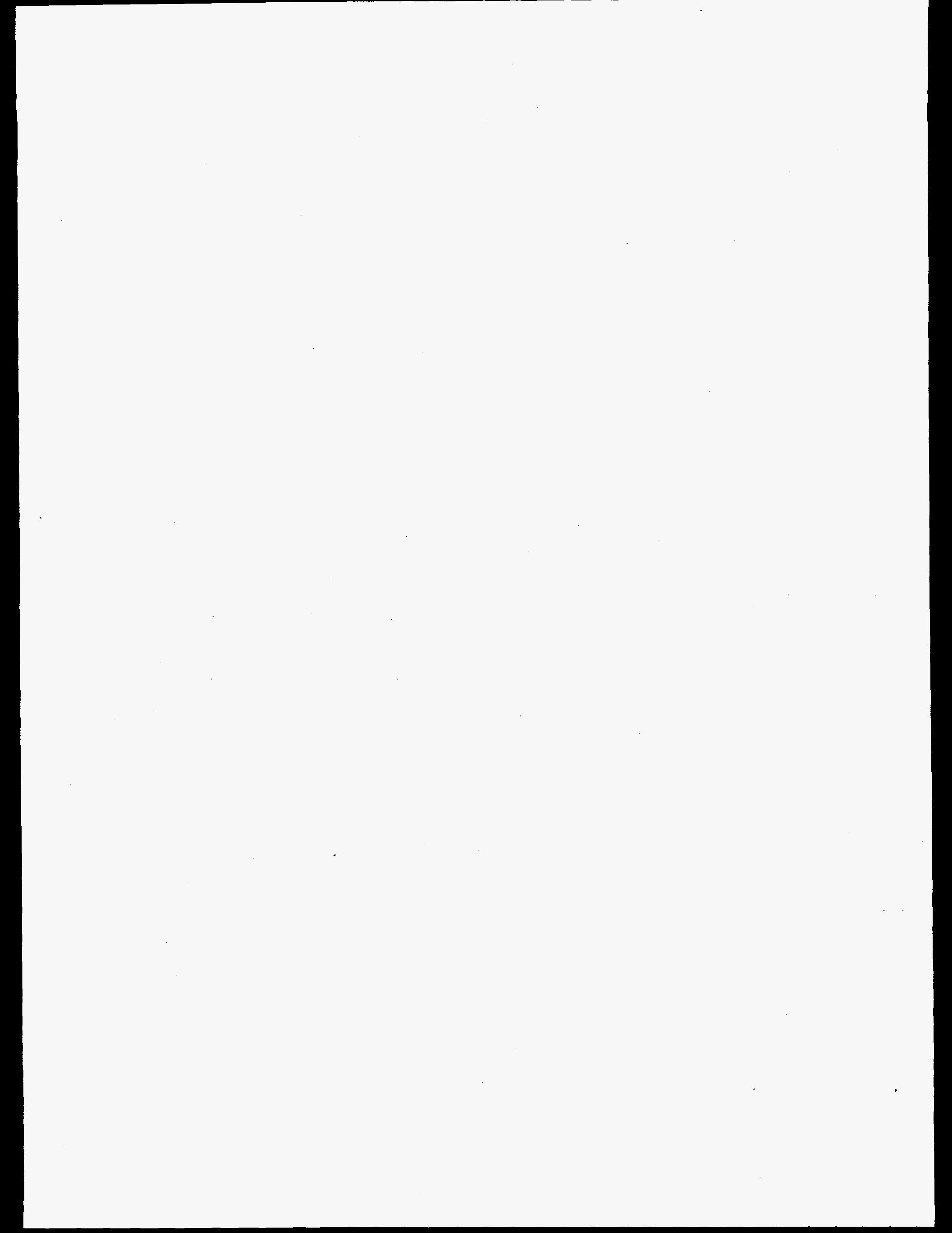


PART 2

CONTENT AND FORMAT OF DATA FILES 


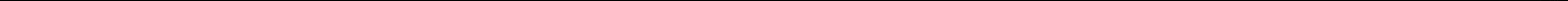




\section{FILE DESCRIPTIONS}

This section describes the content and format of each of the 13 files that comprise this numeric data package (NDP) (Table 2). File names and numbers and brief descriptions of the files follow.

- NDP059.DOC (File 1) describes the details of the database. It contains the text from Sects. 1-7 of this document.

- INVENT.FOR (File 2) contains a FORTRAN 77 program for reading INVENT.DAT (File 6).

- DATA.FOR (File 3) contains a FORTRAN 77 program for reading any of the snow depth data files: SNOWDPTH.DAT, WEST.DAT, HPLAINS.DAT, SOUTH.DAT, MIWWST.DAT, SEAST.DAT, and NEAST.DAT (Files 7-13).

- INVENT.SAS (File 4) contains a SAS ${ }^{\circledR}$ program for reading INVENT.DAT (File 6).

- DATA.SAS (File 5) contains a $S A S^{\circledR}$ program for reading any of the snow depth data files.

- INVENT.DAT (File 6) contains the state abbreviation, station name, NCDC station number, WBAN station number, latitude, longitude, elevation, beginning year of record, ending year of record, and a data completeness flag for each station record.

- SNOWDPTH.DAT (File 7) contains daily snow depth data for 388 unique sampling sites (195 NWS "first-order" stations). For the user's convenience, we have also divided the complete snow depth data set into six regional data sets that are listed as follows. These geographic groupings parallel regions associated with the NWS's six Regional Climate Centers (RCCs), ${ }^{1}$ which were established to meet local and regional needs for climate information (see Fig. 2). The extended file descriptions given later in this section provide detailed descriptions of the regions.

- WEST.DAT (File 8) contains daily snow depth data for 41 NWS first order-stations in the Western RCC.

- HPLAINS.DAT (File 9) contains daily snow depth data for 21 NWS first order-stations in the High Plains RCC.

- SOUTH.DAT (File 10) contains daily snow depth data for 29 NWS first order-stations in the Southern RCC.

- MIDWEST.DAT (File 11) contains daily snow depth data for 45 NWS first order-stations in the Midwestern RCC.

- SEAST.DAT (File 12) contains daily snow depth data for 27 NWS first order-stations in the Southeast RCC.

- NEAST.DAT (File 13) contains daily snow depth data for 32 NWS first order-stations in the Northeast RCC.

${ }^{1}$ On the World Wide Web, see the URL http://nic.fb4.noaa.gov/products/regional_climate/index.html 
Table 2. Size and format information for the NDP files

File number and description ${ }^{a}$

File size ${ }^{b}$

(kB)

66.56

0.74

1.86

54

80

FORTRAN 77 program for reading

any of the snow depth data files.

4. INVENT.SAS:

SAS ${ }^{\oplus}$ program for reading

INVENT.DAT.

5. DATA.SAS:

SAS ${ }^{\otimes}$ program for reading

41

80

any of the snow depth data files.

6. INVENT.DAT:

27.16

Contains station location data and period of record information for each of the 195 NWS first-order stations (388 unique sampling sites).

7. SNOWDPTH.DAT:

Contains daily snow depth data for all stations in the database: 195 NWS first-order stations (388 unique sampling sites).

8. WEST.DAT:

Contains daily snow depth data for 41 first-order stations (81 unique sampling sites) in the Western RCC.

9. HPLAINS.DAT:

Contains daily snow depth data for 21 first-order stations (43 unique sampling sites) in the High Plains RCC. 
Table 2 (continued)

File number and description ${ }^{a}$

File size $e^{b}$

(kB)

Logical

Record

records

length

10. SOUTH.DAT:

5,920

21,844

270

Contains daily snow depth data for

29 first-order stations (56 unique

sampling sites) in the Southern RCC.

11. MIDWEST.DAT:

9,358

34,532

270

Contains daily snow depth data for

45 first-order stations (94 unique

sampling sites) in the Midwestern RCC.

12. SEAST.DAT:

Contains daily snow depth data for

27 first-order stations (51 unique

sampling sites) in the Southeast RCC.

13. NEAST.DAT:

5,979

22,061

Contains daily snow depth data for

32 first-order stations (63 unique

sampling sites) in the Northeast RCC.

${ }^{\sigma} \mathrm{RCC}=$ Regional Climate Center

${ }^{b}$ Approximate size of files in noncompressed form. Files in CDIAC's anonymous $\mathrm{ftp}$ area are compressed and are $-6 \%$ this size. 


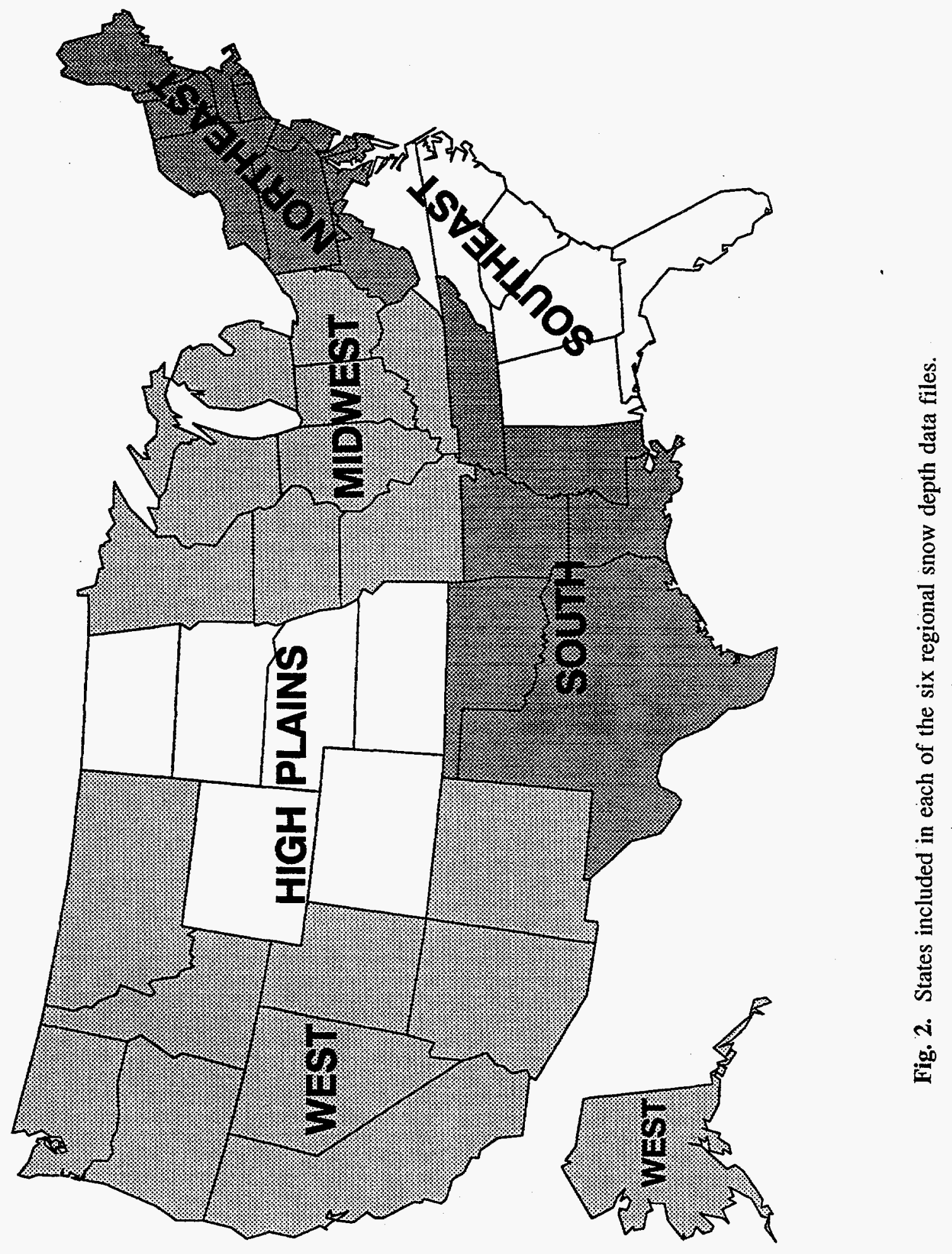




\section{NDP059.DOC (FME 1)}

This file provides a detailed description of the database. It is an electronic version of Sects. 1-7 of this document (figures excluded).

\section{INVENT.FOR (FILE 2)}

This file contains the FORTRAN 77 program to read INVENT.DAT (File 6). The following is a listing of the program.

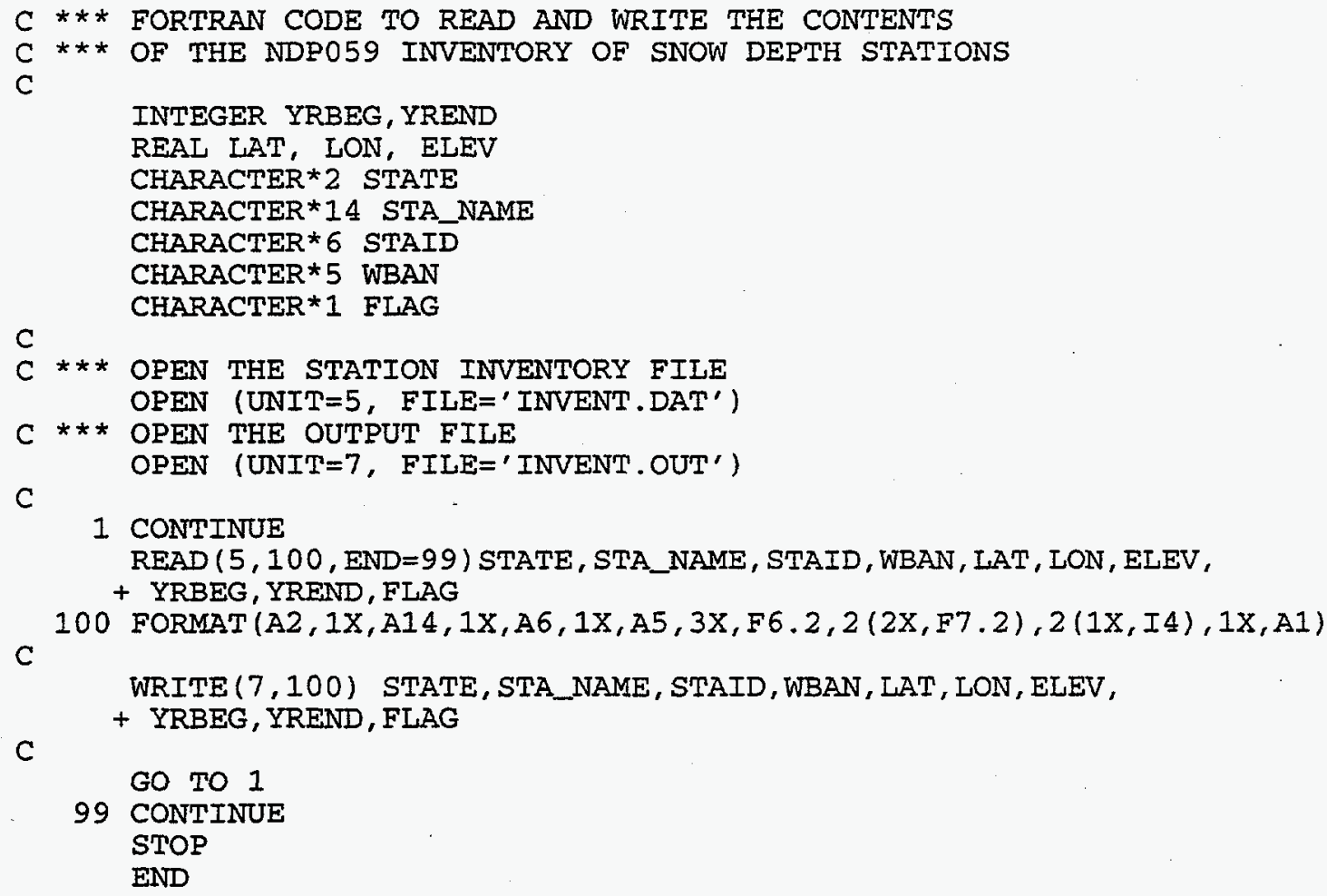

\section{DATA.FOR (FILE 3)}

This file contains the FORTRAN 77 program to read any of the snow depth data files: SNOWDPTH.DAT, WEST.DAT, HPLAINS.DAT, SOUTH.DAT, MIDWEST.DAT, SEAST.DAT, and NEAST.DAT. The following is a listing of the program.

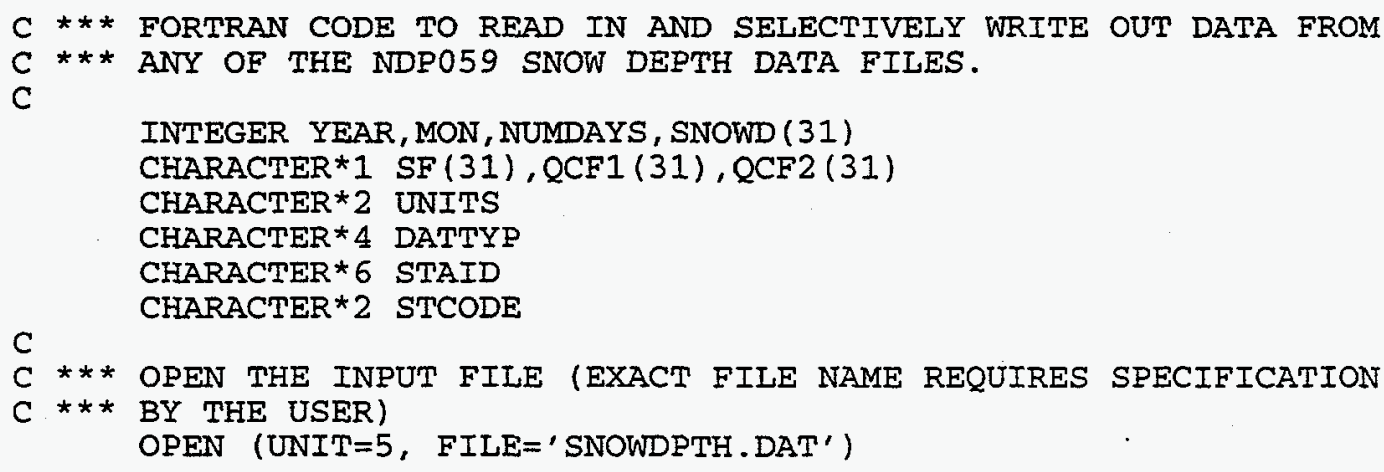




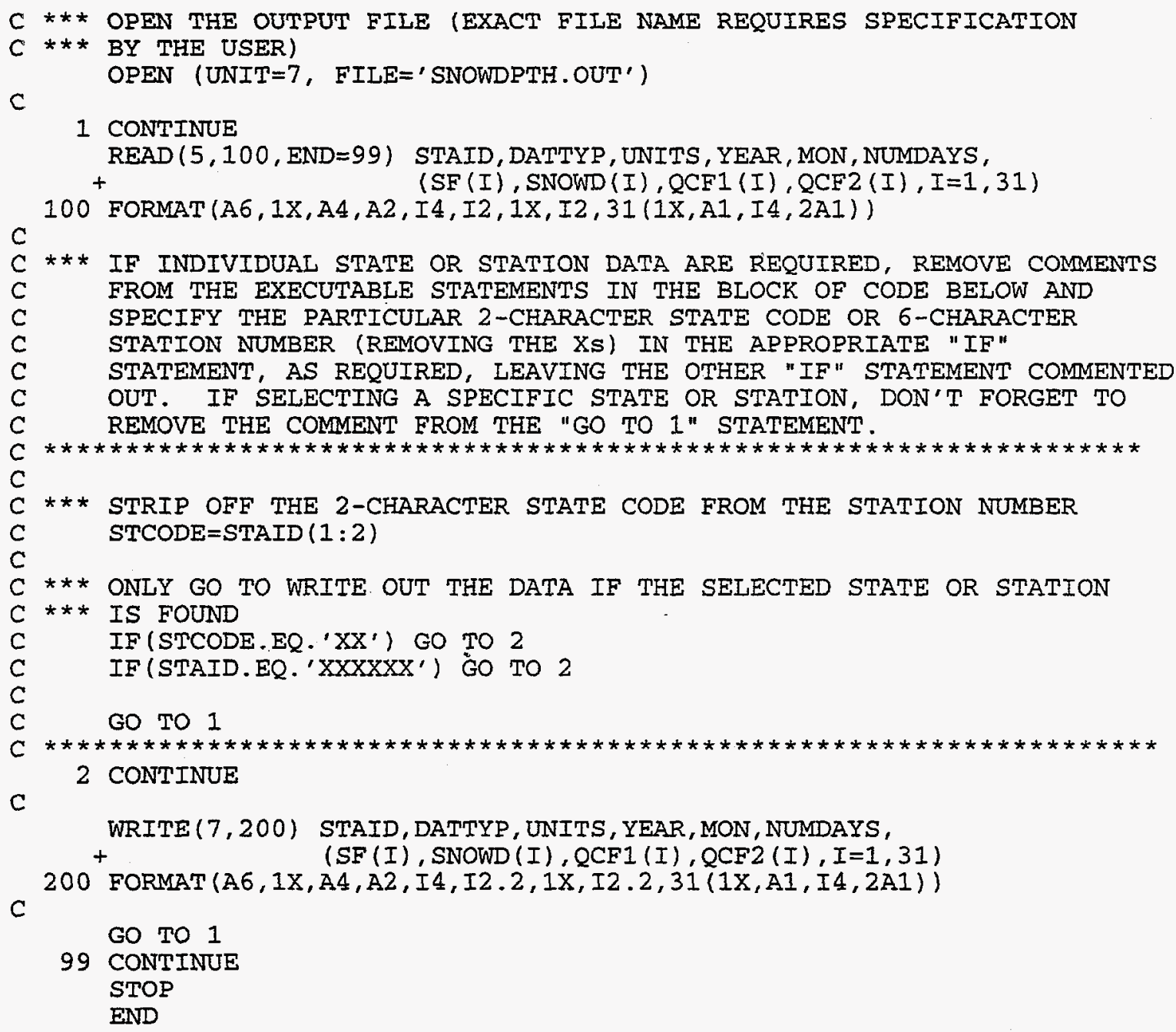

\section{INVENT.SAS (FILE 4)}

This file contains the SAS ${ }^{\oplus}$ program to read INVENT.DAT (File 6). The following is a listing of the program.

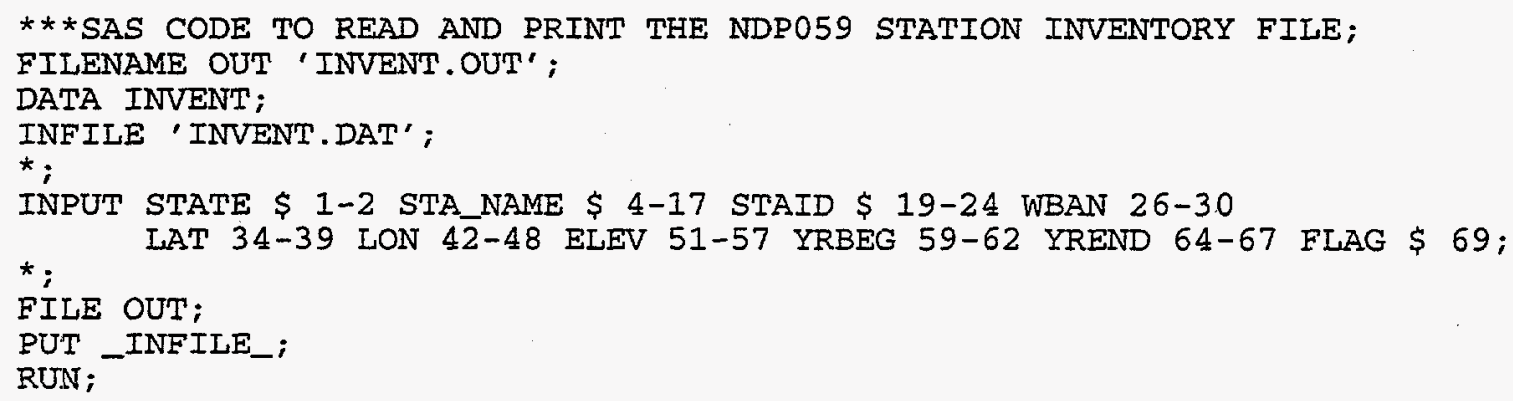




\section{DATA.SAS (FILE 5)}

This file contains the SAS ${ }^{\circledast}$ program to read the snow depth data sets. The following is a listing of the program.

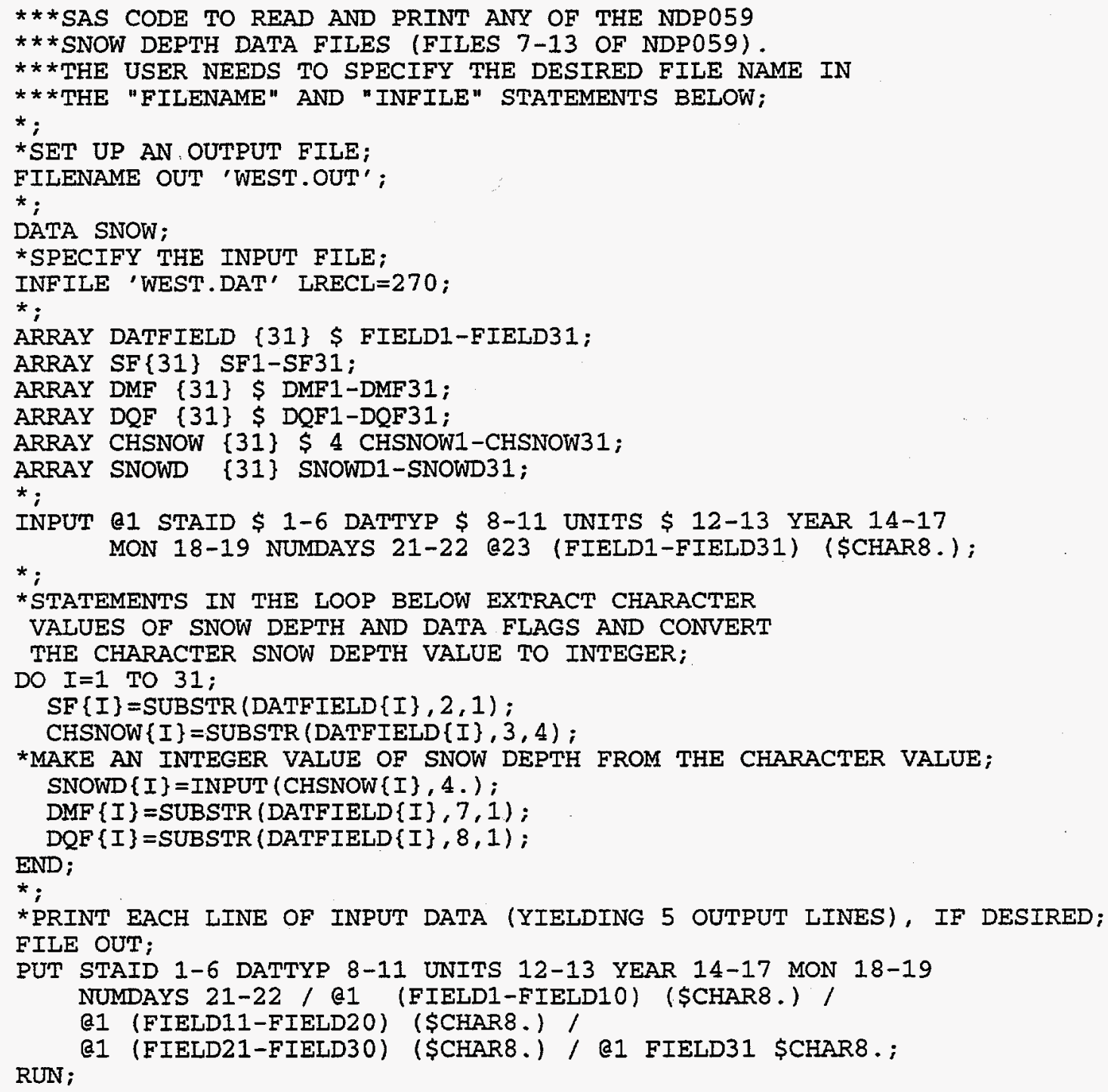

\section{INVENT.DAT (FILE 6)}

The station inventory file was developed by CDIAC from several sources. The NCDC station number was extracted from the snow depth database, and the WBAN number, state abbreviation, and station name were manually entered from the documentation provided by NCDC. The file was then merged with files from Steurer and Karl (1991) in order to add latitude and longitude information. Period of record data were determined through examination of snow depth records. Record completeness flags were defined after a close examination of the number of years and months in each station's record. Elevation data for some stations were added from NCDC's U.S. Historical Climatology Network (U.S. HCN) database (Easterling et al. 1996). A list of stations with missing latitude, longitude, and 
elevation data was sent to NCDC for their input. After these data were received and manually entered into the database, latitude and longitude values were converted from degrees and minutes to decimal degrees, and elevation values were converted to meters. A final list of stations with missing or questionable values was sent to NCDC; the data returned were then entered into the station inventory file.

The station inventory file contains the state abbreviation, station name, NCDC station number, WBAN station number, latitude, longitude, elevation above sea level, beginning year of record, ending year of record, and a flag denoting the completeness of the station record for all 388 unique snow depth sampling locations. Stated in tabular form, the contents of the station inventory file include the following.

\begin{tabular}{llccr}
\hline Variable & $\begin{array}{l}\text { Variable } \\
\text { type }\end{array}$ & $\begin{array}{c}\text { Variable } \\
\text { width }\end{array}$ & $\begin{array}{c}\text { Starting } \\
\text { column }\end{array}$ & $\begin{array}{r}\text { Ending } \\
\text { column }\end{array}$ \\
\hline STATE & Character & 2 & 1 & 2 \\
STA_NAME & Character & 14 & 4 & 17 \\
STAID & Alphanumeric & 6 & 19 & 24 \\
WBAN & Character & 5 & 26 & 30 \\
LAT & Numeric & 6 & 34 & 39 \\
LON & Numeric & 7 & 42 & 48 \\
ELEV & Numeric & 7 & 51 & 57 \\
YRBEG & Numeric & 4 & 59 & 62 \\
YREND & Numeric & 4 & 64 & 67 \\
FLAG & Character & 1 & 69 & 69 \\
\hline
\end{tabular}

The variables are defined as follows:

STATE is the two-letter state abbreviation (See Appendix A for a listing of the state abbreviations, their associated two-digit NCDC state code, and the state name.);

STA_NAME is the station name, usually a city;

STAID is the unique NCDC station code formed by combining the two-digit NCDC state number with the four-digit Cooperative Network Index number assigned by NCDC. The code is defined as alphanumeric to allow for character station codes (e.g., 50OMEV for Nome, Alaska) as well as to preserve leading zeros upon output;

WBAN is the Weather Bureau Army Navy Network index, defined as character to allow for preserving leading zeros upon output (missing values are represented by 99999);

LAT is the latitude of the station in decimal degrees north (missing values are represented by -99.99 ); 
LON is the longitude of the station in decimal degrees west (missing values are represented by -99.99 );

ELEV is the elevation of the station above sea level in meters (missing values are represented by -99.99 ). Values range from 1.00 to 2132.00 ;

YRBEG is the beginning year of the station's period of record. Values range from 1893 to 1979 ;

YREND is the ending year of the station's period of record. Values range from 1896 to 1992 ;

FLAG is the record-completeness flag that grossly characterizes the completeness of the data. Possible values of FLAG are:

$A=$ There are seven or more months of data in every year of the particular station number's record.

$\mathrm{B}=$ The first year or two of the record is missing one or two months of data, or one or two years in the middle or at the end of the record is missing one month of data; the remaining years in the station record have seven or more months of data.

$\mathrm{C}=$ Several years are missing one to three months of data.

$\mathrm{D}=$ Many years are missing at least one and usually many months of data.

$\mathrm{E}=$ One or more years are missing in the station record.

$F=$ The one-year station record has seven months of winter season data.

$\mathrm{G}=$ The one-year station record has less than seven months of winter season data.

\section{SNOWDPTH.DAT (FILE 7)}

This file contains daily snow depth data for 195 NWS first-order stations (388 unique sampling sites) in 48 states; no observations from Delaware or Hawaii are included in the database. The file is sorted by the six-digit NCDC station number, year, and month. Each data record contains one month's worth of daily snow depth values and includes the NCDC station number, data type, units, year, month, number of days in the month, and 31 daily snow depth data values with their respective flags. Stated in tabular form, the contents include the following variables. 


\begin{tabular}{llccc}
\hline Variable & $\begin{array}{l}\text { Variable } \\
\text { type }\end{array}$ & $\begin{array}{c}\text { Variable } \\
\text { width }\end{array}$ & $\begin{array}{c}\text { Starting } \\
\text { column }\end{array}$ & $\begin{array}{c}\text { Ending } \\
\text { column }^{a}\end{array}$ \\
\hline STAID & Alphanumeric & 6 & 1 & 6 \\
DATTYP & Character & 4 & 8 & 11 \\
UNITS & Character & 2 & 12 & 13 \\
YEAR & Numeric & 4 & 14 & 17 \\
MON & Numeric & 2 & 18 & 19 \\
NUMDAYS & Numeric & 2 & 21 & 22 \\
SF(1) & Alphanumeric & 1 & 24 & 28 \\
SNOWD(1) & Numeric & 4 & 25 & 29 \\
DMF(1) & Alphanumeric & 1 & 29 & 30 \\
DQF(1) & Alphanumeric & 1 & 30 & $*$ \\
SF(2-31) & Alphanumeric & 1 & $*$ & $*$ \\
SNOWD(2-31) & Numeric & 4 & $*$ & $*$ \\
DMF(2-31) & Alphanumeric & 1 & $*$ & $*$ \\
DQF(2-31) & Alphanumeric & 1 & & 28 \\
& & & & $*$ \\
\hline
\end{tabular}

${ }^{a}$ Values for entries filled with an asterisk may be obtained using: $\operatorname{COL}(\mathrm{N})=\operatorname{COL}(1)+(\mathrm{N} \times 8)-8$, where $\operatorname{COL}(\mathrm{N})$ is the starting/ending column for SF(N), SNOWD(N), DMF(N), or DQF(N); COL(1) is the starting/ending column for $\mathrm{SF}(1)$, SNOWD(1), DMF(1), or DQF(1); and $\mathrm{N}$ is the day of the month (2-31).

The variables are defined as follows:

STAID is the unique NCDC station code formed by combining the two-digit NCDC state number with the four-digit Cooperative Network Index number assigned by NCDC. The code is defined as alphanumeric to allow for character station codes (e.g., 50OMEV for Nome, Alaska) as well as to preserve leading zeros upon output;

DATTYP

is the data type (SNWD = snow depth);

UNITS

is the units of the snow depth data ( $T I=$ tenths of inches);

YEAR

is the year of the data record;

MON

is the month of the data record;

NUMDAYS

is the number of days in the month;

$\mathrm{SF}(1-31)$

are the source flags for the daily data values;

SNOWD(1-31) are daily snow depth data values at observation time: 00:30 GMT prior to July $1952 ; 12: 30$ GMT for July 1,1952 , to May 31,1957 ; and 12:00 GMT for June 1, 1957, to present. Missing values are coded as -999 . Hail is included with snowfall from July 1948 to December 1955 and from May 1989 to the present, as described in the on-line documentation of NCDC's Summary of the Day-First Order database (NCDC Tape Deck 3210) (http://www.ncdc.noaa.gov/pub/data/fsod/fsod.README);

DMF(1-31) are the data measurement flags for the daily data values; and 
$\mathrm{DQF}(1-31) \quad$ are the data quality flags for the daily data values.

\section{Flag codes for the SNOW DEPTH data}

is a code indicating the source of the daily data value. The codes and their meanings are as follows:

$0=$ NCDC Tape Deck 3210, Summary of the Day-First Order, element digital file;

3 = Manuscript-original records, National Climatic Data Center;

7 = Local Climatological Data (LCD) (monthly NCDC publication);

Blank $=$ Not applicable [used for nonexistent days of the month (e.g., 30 February)].

DMF

is the data measurement flag, which describes how the daily value was measured. The codes and their meanings are as follows:

$A=$ Accumulated amount. This value is the amount of snow accumulated since the last measurement;

$\mathrm{E}=$ Estimated value;

$L=$ Reflects sleet;

$\mathrm{T}=$ Trace (data value $=0000$ for a trace); and

Blank = Valid original data (no flag needed).

DQF is the data quality flag. In January 1982, NCDC instituted a computer algorithm for automated validation of digital data archives. The system checks the internal consistency of a station's data and compares each station's observations to prescribed climatological limits and observations from surrounding stations. Numeric DQF codes apply only to NCDC's digital data, i.e., where the source flag (SF) is equal to "0" for a particular value. Alphabetic codes describe the particular manual or automated NCDC procedure employed to correct or estimate a data value. The codes and their meanings are as follows:

$0=$ Valid data (observed data have passed all internal consistency checks);

1 = Validity indeterminable (used primarily for pre-1984 data);

3 = Data beginning January 1, 1984: observed data have failed an internal consistency check, for example, the snow depth value is inconsistent with the water equivalent precipitation observation. No edited value follows, or value may have been set equal to zero. Data prior to January 1, 1984: observed data failed a climatological limits check. In other words, the value was physically impossible. No edited value follows, or value may have been set equal to zero.;

$4=$ Observed data value invalid, for example, negative or extremely large value. No edited value follows, or value may have been set equal to zero;

$\mathrm{E}=$ Edited data value passes all system's checks (no observed value present); 
$\mathrm{S}=$ Manually edited value passes all system's checks; and

Blank = Flag not needed.

For the user's convenience, the complete snow depth data set (SNOWDPTH.DAT) has been divided into six regional data sets that are listed below. These geographic groupings parallel regions associated with the National Weather Service's six Regional Climate Centers, which were established to meet local and regional needs for climate information. The file descriptions include the states as well as the number of stations within each region.

\section{WEST.DAT (FILE 8)}

This file contains daily snow depth data for 41 NWS first-order stations (81 unique sampling locations) in the Western RCC. States included in this region are Washington, Oregon, Idaho, Montana, California, Nevada, Utah, Arizona, New Mexico, and Alaska.

\section{HPLAINS.DAT (FILE 9)}

This file contains daily snow depth data for 21 NWS first-order stations (43 unique sampling locations) in the High Plains RCC. States included in this region are North Dakota, South Dakota, Nebraska, Kansas, Wyoming, and Colorado.

\section{SOUTH.DAT (FILE 10)}

This file contains daily snow depth data for 29 NWS first order-stations (56 unique sampling locations) in the Southern RCC. States included in this region are Tennessee, Mississippi, Arkansas, Louisiana, Oklahoma, and Texas.

\section{MDWEST.DAT (FILE 11)}

This file contains daily snow depth data for 45 NWS first order-stations (94 unique sampling locations) in the Midwestern RCC. States included in this region are Minnesota, Wisconsin, Michigan, Iowa, Missouri, Mlinois, Indiana, Ohio, and Kentucky.

\section{SEAST.DAT (FILE 12)}

This file contains daily snow depth data for 27 NWS first order-stations (51 unique sampling locations) in the Southeast RCC. States included in this region are Virginia, North Carolina, South Carolina, Georgia, Alabama, and Florida.

\section{NEAST.DAT (FILE 13)}

This file contains daily snow depth data for 32 NWS first order-stations (63 unique sampling locations) in the Northeast RCC. States included in this region are Maine, New Hampshire, Vermont, Massachusetts, Connecticut, Rhode Island, New York, Pennsylvania, New Jersey, Maryland, and West Virginia. No snow depth observations are included from Delaware. 


\section{REFERENCE}

Easterling, D. R., T. R. Karl, E. H. Mason, P. Y. Hughes, and D. P. Bowman. 1996. United States Historical Climatology Network (U. S. HCN) Monthly Temperature and Precipitation Data. R. C. Daniels and T. A. Boden (eds). ORNL/CDIAC-87, NDP-019/R3. Carbon Dioxide Information Analysis Center, Oak Ridge National Laboratory, Oak Ridge, Tennessee.

\section{VERIFICATION OF DATA TRANSPORT}

The data files contained in this NDP can be read by using the FORTRAN 77 and SAS ${ }^{\circledR}$ programs provided. Users should verify that the data have been correctly transported to their systems by visually examining each data file, where practical. To facilitate the visual inspection process, partial listings of each data file are provided in Tables 3 and 4 . Each of these tables contains the first five and last five records of the data file.

Table 3. Partial listing of the file invent.dat (File 6)

First five records of the file:

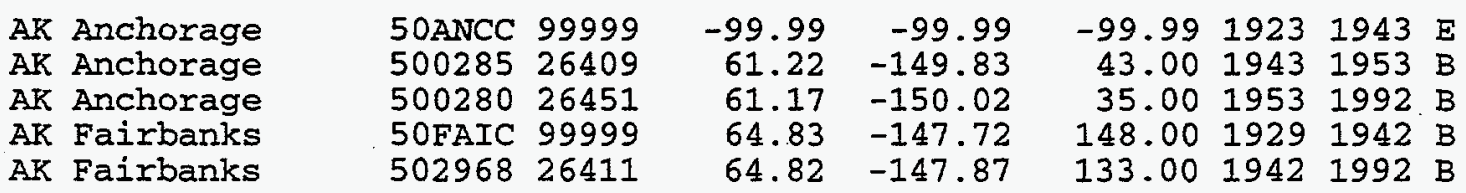

Last five records of the file:

WY Cheyenne
WY Lander
WY Lander
WY Sheridan
WY Sheridan

48167524018

48INDC 24075

$485390 \quad 24021$

48SHRC 24058

$488155 \quad 24029$

$\begin{array}{llllll}41.15 & -104.82 & 1866.00 & 1935 & 1992 & \mathrm{~B} \\ 42.83 & -108.73 & 1631.00 & 1893 & 1946 & \mathrm{C} \\ 42.82 & -108.73 & 1637.00 & 1946 & 1992 & \mathrm{~B} \\ 44.80 & -106.95 & 1150.00 & 1907 & 1940 & \mathrm{~B} \\ 44.77 & -106.97 & 1209.00 & 1940 & 1992 & \mathrm{~B}\end{array}$


Table 4. Partial listings of the snow depth files (Files 7-13)

\section{SNOWDPTH.DAT (FILE 7):}

First five records of the file:

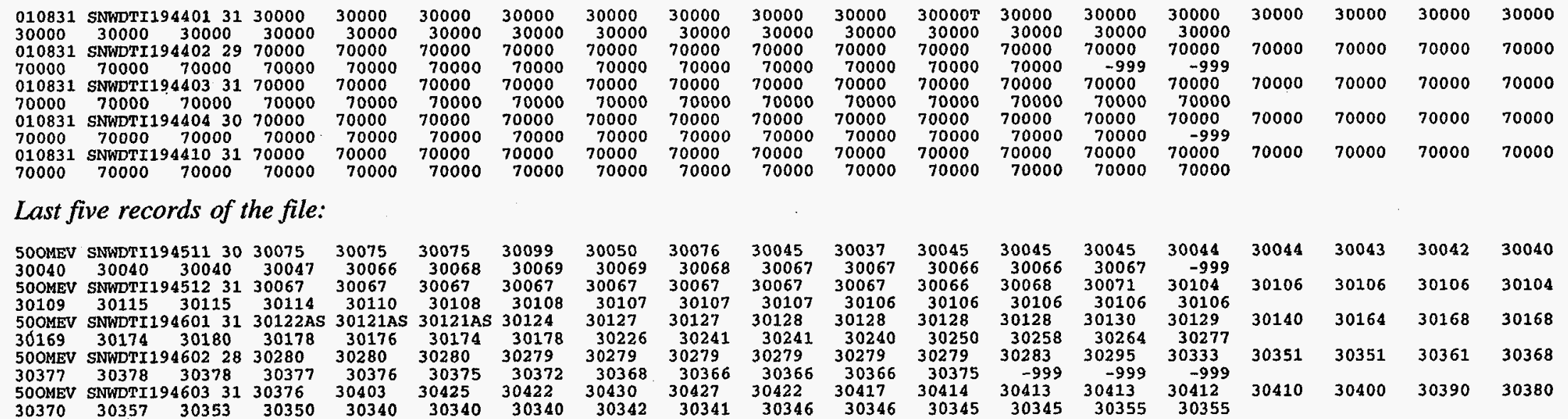

\section{WEST.DAT (FILE 8):}

First five records of the file:

\begin{tabular}{|c|c|c|c|c|c|c|c|c|c|c|c|c|c|c|c|c|c|c|}
\hline $\begin{array}{l}023007 \\
30000 \\
023007 \\
70000 \\
023007 \\
30000 \\
023007 \\
30030 \\
023007 \\
30000\end{array}$ & $\begin{array}{l}\text { SNWDTI18 } \\
30000 \\
\text { SNWDTI18 } \\
70000 \\
\text { SNWDTI18 } \\
30000 \\
\text { SNWDTI118 } \\
30094 \\
\text { SNWDTI19 } \\
30000\end{array}$ & $\begin{array}{c}8990531 \\
30000 \\
8991031 \\
70000 \\
8991130 \\
30000 \\
8991231 \\
30000 \\
9000131 \\
30000\end{array}$ & $\begin{array}{c}30000 \mathrm{~T} \\
30000 \\
70000 \\
70000 \\
30000 \\
30000 \\
30000 \\
30000 \\
30000 \\
30000\end{array}$ & $\begin{array}{c}30004 \\
30000 \\
70000 \\
70000 \\
30000 \\
30000 \\
30000 \\
30000 \\
30000 \\
30000\end{array}$ & $\begin{array}{c}30000 \\
30000 \\
70000 \\
70000 \\
30000 \\
30094 \\
30000 \\
30000 \\
30000 \mathrm{~T} \\
30000\end{array}$ & $\begin{array}{c}30000 \\
30000 \\
70000 \\
70000 \\
30000 \\
30000 \\
30000 \\
30000 \\
30000 \mathrm{~T} . \\
30000\end{array}$ & $\begin{array}{c}30000 \\
30000 \\
70000 \\
70000 \\
30000 \\
30000 \\
30000 \\
30000 \\
30000 \\
30000\end{array}$ & $\begin{array}{c}30000 \\
30000 \\
70000 \\
70000 \\
30000 \\
30000 \\
30000 \% \\
30000 \\
30000 \\
30000\end{array}$ & $\begin{array}{c}30000 \\
30000 \\
70000 \\
70000 \\
30000 \\
30000 \\
30000 \\
30000 \\
30000 \\
30000\end{array}$ & $\begin{array}{c}30000 \\
30000 \\
70000 \\
70000 \\
30000 \\
30000 \\
30000 \\
30000 \\
30000 \mathrm{~T} \\
30000\end{array}$ & $\begin{array}{c}30000 \\
30000 \\
70000 \\
70000 \\
30000 \\
30000 \\
30000 \mathrm{~T} \\
30000 \\
30002 \\
30000\end{array}$ & $\begin{array}{c}30000 \\
30000 \\
70000 \\
70000 \\
30000 \\
30000 \\
30000 \\
30000 \\
30000 \\
30000\end{array}$ & $\begin{array}{c}30000 \\
30000 \\
70000 \\
70000 \\
30000 \\
30000 \\
30000 \\
30000 \mathrm{~T} \\
30000 \\
30000\end{array}$ & $\begin{array}{c}30000 \\
30000 \\
70000 \\
70000 \\
30000 \\
-999 \\
30000 \\
30000 \\
30000 \\
30000\end{array}$ & 30000 & 30000 & $30000 \mathrm{~T}$ & $\begin{array}{l}30000 \\
30000\end{array}$ \\
\hline \multicolumn{19}{|c|}{ Last five records of the file: } \\
\hline $\begin{array}{l}500 \mathrm{MEV} \\
30040 \\
500 \mathrm{MEV} \\
30109 \\
500 \mathrm{MEV} \\
30169 \\
500 \mathrm{MEV} \\
30377 \\
500 \mathrm{MEV} \\
30370\end{array}$ & $\begin{array}{l}\text { SNWDTI19 } \\
30040 \\
\text { SNWDTI19 } \\
30115 \\
\text { SNWDTI19 } \\
30174 \\
\text { SNWDTI19 } 19 \\
30378 \\
\text { SNWDTI19 } \\
30357\end{array}$ & $\begin{array}{c}9451130 \\
30040 \\
9451231 \\
30115 \\
9460131 \\
30180 \\
9460228 \\
30378 \\
9460331 \\
30353\end{array}$ & $\begin{array}{c}30075 \\
30047 \\
30067 \\
30114 \\
30122 \mathrm{AS} \\
30178 \\
30280 \\
30377 \\
30376 \\
30350\end{array}$ & $\begin{array}{c}30075 \\
30066 \\
30067 \\
30110 \\
30121 \mathrm{AS} \\
30176 \\
30280 \\
30376 \\
30403 \\
30340\end{array}$ & $\begin{array}{c}30075 \\
30068 \\
30067 \\
30108 \\
30121 \mathrm{AS} \\
30174 \\
30280 \\
30375 \\
30425 \\
30340\end{array}$ & $\begin{array}{c}30099 \\
30069 \\
30067 \\
30108 \\
30124 \\
30178 \\
30279 \\
30372 \\
30422 \\
30340\end{array}$ & $\begin{array}{c}30050 \\
30069 \\
30067 \\
30107 \\
30127 \\
30226 \\
30279 \\
30368 \\
30430 \\
30342\end{array}$ & $\begin{array}{c}30076 \\
30068 \\
30067 \\
30107 \\
30127 \\
30241 \\
30279 \\
30366 \\
30427 \\
30341\end{array}$ & $\begin{array}{c}30045 \\
30067 \\
30067 \\
30107 \\
30128 \\
30241 \\
30279 \\
30366 \\
30422 \\
30346\end{array}$ & $\begin{array}{c}30037 \\
30067 \\
30067 \\
30106 \\
30128 \\
30240 \\
30279 \\
30366 \\
30417 \\
30346\end{array}$ & $\begin{array}{c}30045 \\
30066 \\
30066 \\
30106 \\
30128 \\
30250 \\
30279 \\
30375 \\
30414 \\
30345\end{array}$ & $\begin{array}{c}30045 \\
30066 \\
30068 \\
30106 \\
30128 \\
30258 \\
30283 \\
-999 \\
30413 \\
30345\end{array}$ & $\begin{array}{c}30045 \\
30067 \\
30071 \\
30106 \\
30130 \\
30264 \\
30295 \\
-999 \\
30413 \\
30355\end{array}$ & $\begin{array}{c}30044 \\
-999 \\
30104 \\
30106 \\
30129 \\
30277 \\
30333 \\
-999 \\
30412 \\
30355\end{array}$ & $\begin{array}{l}30106 \\
30140\end{array}$ & $\begin{array}{l}30106 \\
30164\end{array}$ & $\begin{array}{l}30106 \\
30168\end{array}$ & $\begin{array}{l}30040 \\
30104 \\
30168 \\
30368\end{array}$ \\
\hline
\end{tabular}


Table 4 (continued)

\section{HPLAINS.DAT (FILE 9):}

First five records of the file:

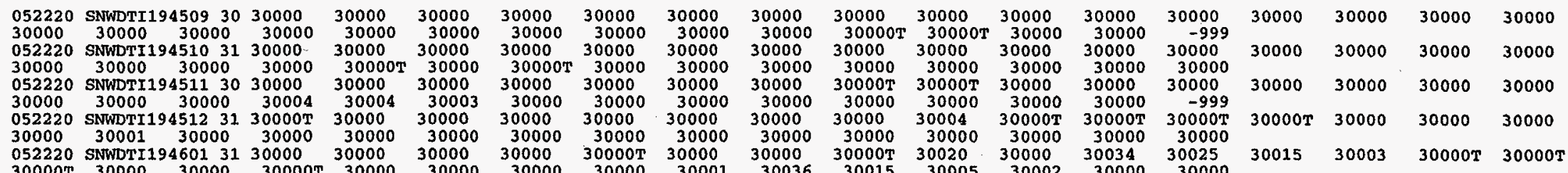

Last five records of the file:

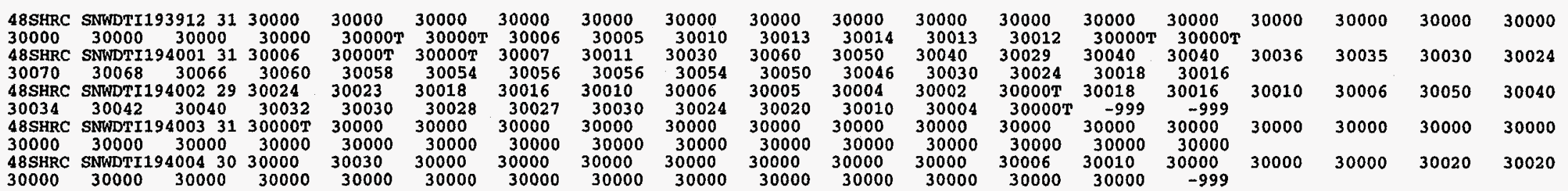

\section{SOUTH.DAT (FILE 10):}

First five records of the file:

\begin{tabular}{|c|c|c|c|c|c|c|c|c|c|c|c|c|c|c|c|c|c|c|}
\hline $\begin{array}{l}032574 \\
70000 \\
032574 \\
70000 \\
032574 \\
30000 T \\
032574 \\
70000 \\
032574 \\
70000\end{array}$ & $\begin{array}{l}\text { SNWDTI19 } \\
70000 \\
\text { SNWDTI19 } \\
70000 \\
\text { SNWDTI19 } \\
30005 \\
\text { SNWDTI19 } \\
70000 \\
\text { SNWDTI19 } \\
70000\end{array}$ & $\begin{array}{c}9451031 \\
70000 \\
9451130 \\
70000 \\
9451231 \\
30000 \\
9460131 \\
70000 \\
9460331 \\
70000\end{array}$ & $\begin{array}{c}70000 \\
70000 \\
70000 \\
70000 \\
30000 \\
30000 \\
70000 \\
70000 \\
70000 \\
70000\end{array}$ & $\begin{array}{c}70000 \\
70000 \\
70000 \\
70000 \\
30000 \\
30000 \\
70000 \\
70000 \\
70000 \\
70000\end{array}$ & $\begin{array}{c}70000 \\
70000 \\
70000 \\
70000 \\
30000 \\
30000 \\
70000 \\
70000 \\
70000 \\
70000\end{array}$ & $\begin{array}{c}70000 \\
70000 \\
70000 \\
70000 \\
30000 \\
30000 \\
70000 \\
70000 \\
70000 \\
70000\end{array}$ & $\begin{array}{c}70000 \\
70000 \\
70000 \\
70000 \\
30000 \\
30000 \\
70000 \\
70000 \\
70000 \\
70000\end{array}$ & $\begin{array}{c}70000 \\
70000 \\
70000 \\
70000 \\
30000 \\
30000 \\
70000 \\
70000 \\
70000 \\
70000\end{array}$ & $\begin{array}{c}70000 \\
70000 \\
70000 \\
70000 \\
30000 \\
30000 \\
70000 \\
70000 \\
70000 \\
70000\end{array}$ & $\begin{array}{c}70000 \\
70000 \\
70000 \\
70000 \\
30000 \\
30000 \\
70000 \\
70000 \\
70000 \\
70000\end{array}$ & $\begin{array}{c}70000 \\
70000 \\
70000 \\
70000 \\
30000 \\
30000 \\
70000 \\
70000 \\
70000 \\
70000\end{array}$ & $\begin{array}{c}70000 \\
70000 \\
70000 \\
70000 \\
30000 \\
30000 \\
70000 \\
70000 \\
70000 \\
70000\end{array}$ & $\begin{array}{c}70000 \\
70000 \\
70000 \\
70000 \\
30000 \\
30000 \\
70000 \\
70000 \\
70000 \\
70000\end{array}$ & $\begin{array}{c}70000 \\
70000 \\
70000 \\
-999 \\
30000 \\
30000 \\
70000 \\
70000 \\
70000 \\
70000\end{array}$ & 30000 & 30000 & $\begin{array}{l}70000 \\
30000 \\
70000\end{array}$ & $\begin{array}{l}70000 \\
30000\end{array}$ \\
\hline \multicolumn{19}{|c|}{ Last five records of the file: } \\
\hline $\begin{array}{l}\text { 41SATC } \\
70000 \\
41 \text { SATC } \\
70000 \\
41 \text { SATC } \\
70000 \\
41 \text { SATC } \\
\text { 70000 } \\
\text { 41SATC } \\
\text { 70000 }\end{array}$ & $\begin{array}{l}\text { SNWDTI19 } \\
70000 \\
\text { SNWDTI19 } \\
70000 \\
\text { SNWDTI19 } \\
70000 \\
\text { SNWDTI19 } \\
70000 \\
\text { SNWDTI19 } \\
70000\end{array}$ & $\begin{array}{c}9003 \quad 31 \\
70000 \\
9400430 \\
70000 \\
9401031 \\
70000 \\
901130 \\
70000 \\
901231 \\
70000\end{array}$ & $\begin{array}{c}70000 \\
70000 \\
70000 \\
70000 \\
70000 \\
70000 \\
70000 \\
70000 \\
70000 \\
70000\end{array}$ & $\begin{array}{c}70000 \\
70000 \\
70000 \\
70000 \\
70000 \\
70000 \\
70000 \\
70000 \\
70000 \\
70000\end{array}$ & $\begin{array}{c}70000 \\
70000 \\
70000 \\
70000 \\
70000 \\
70000 \\
70000 \\
70000 \\
70000 \\
70000\end{array}$ & $\begin{array}{c}70000 \\
70000 \\
70000 \\
70000 \\
70000 \\
70000 \\
70000 \\
70000 \\
70000 \\
70000\end{array}$ & $\begin{array}{c}70000 \\
70000 \\
70000 \\
70000 \\
70000 \\
70000 \\
70000 \\
70000 \\
70000 \\
70000\end{array}$ & $\begin{array}{c}70000 \\
70000 \\
70000 \\
70000 \\
70000 \\
70000 \\
70000 \\
70000 \\
70000 \\
70000\end{array}$ & $\begin{array}{c}70000 \\
70000 \\
70000 \\
70000 \\
70000 \\
70000 \\
70000 \\
70000 \\
70000 \\
70000\end{array}$ & $\begin{array}{c}70000 \\
70000 \\
70000 \\
70000 \\
70000 \\
70000 \\
70000 \\
70000 \\
70000 \\
70000\end{array}$ & $\begin{array}{c}70000 \\
70000 \\
70000 \\
70000 \\
70000 \\
70000 \\
70000 \\
70000 \\
70000 \\
70000\end{array}$ & $\begin{array}{c}70000 \\
70000 \\
70000 \\
70000 \\
70000 \\
70000 \\
70000 \\
70000 \\
70000 \\
70000\end{array}$ & $\begin{array}{c}70000 \\
70000 \\
70000 \\
70000 \\
70000 \\
70000 \\
70000 \\
70000 \\
70000 \\
70000\end{array}$ & $\begin{array}{c}70000 \\
70000 \\
70000 \\
-999 \\
70000 \\
70000 \\
70000 \\
-999 \\
70000 \\
70000\end{array}$ & $\begin{array}{l}70000 \\
70000\end{array}$ & $\begin{array}{l}70000 \\
70000 \\
70000 \\
70000\end{array}$ & $\begin{array}{l}70000 \\
70000 \\
70000 \\
70000\end{array}$ & $\begin{array}{l}70000 \\
70000 \\
70000 \\
70000\end{array}$ \\
\hline
\end{tabular}


Table 4 (continued)

\section{MIDWEST.DAT (FILE 11):}

First five records of the file:

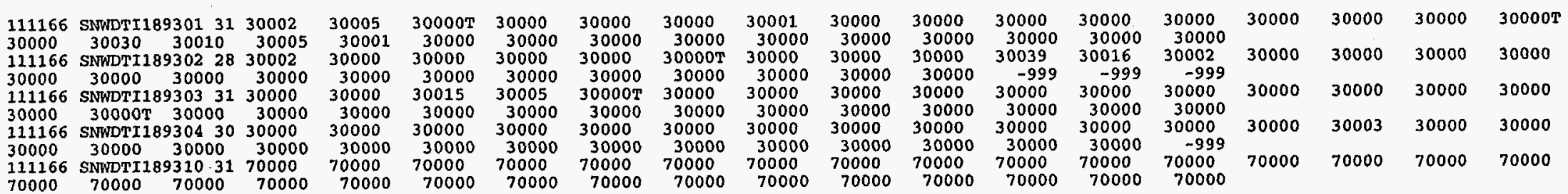

Last five records of the file:

47GRBC SNWDTI1949043000030100010100000T1 00000T1 00000T100000100000100000100000100000100000100000100000100000100020100000T1 00000T1 $00000100000100000100000100000100000100000100000110000010000010000010000010000011-999$

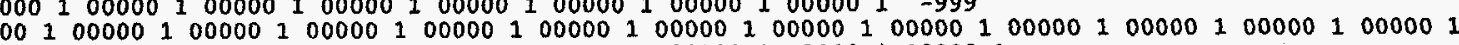
$0000010000010000010000010000010000010000010000010000010000010000010000010000010000011-999$

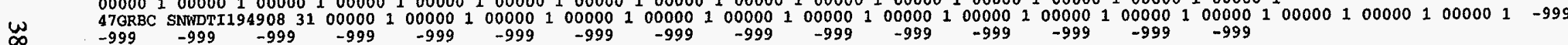

\section{SEAST.DAT (FILE 12):}

First five records of the file:

\begin{tabular}{|c|c|c|c|c|c|c|c|c|c|c|c|c|c|c|c|c|c|c|}
\hline $\begin{array}{l}010831 \\
30000 \\
010831 \\
70000 \\
010831 \\
70000 \\
010831 \\
70000 \\
010831 \\
70000\end{array}$ & $\begin{array}{l}\text { SNWDTI19 } \\
30000 \\
\text { SNWDTI19 } \\
70000 \\
\text { SNWDTI19 } \\
70000 \\
\text { SNWDTI19 } \\
70000 \\
\text { SNWDTI19 } \\
70000\end{array}$ & $\begin{array}{c}440131 \\
30000 \\
440229 \\
70000 \\
440331 \\
70000 \\
440430 \\
70000 \\
441031 \\
70000\end{array}$ & $\begin{array}{c}30000 \\
30000 \\
70000 \\
70000 \\
70000 \\
70000 \\
70000 \\
70000 \\
70000 \\
70000\end{array}$ & $\begin{array}{c}30000 \\
30000 \\
70000 \\
70000 \\
70000 \\
70000 \\
70000 \\
70000 \\
70000 \\
70000\end{array}$ & $\begin{array}{c}30000 \\
30000 \\
70000 \\
70000 \\
70000 \\
70000 \\
70000 \\
70000 \\
70000 \\
70000\end{array}$ & $\begin{array}{c}30000 \\
30000 \\
70000 \\
70000 \\
70000 \\
70000 \\
70000 \\
70000 \\
70000 \\
70000\end{array}$ & $\begin{array}{c}30000 \\
30000 \\
70000 \\
70000 \\
70000 \\
70000 \\
70000 \\
70000 \\
70000 \\
70000\end{array}$ & $\begin{array}{c}30000 \\
30000 \\
70000 \\
70000 \\
70000 \\
70000 \\
70000 \\
70000 \\
70000 \\
70000\end{array}$ & $\begin{array}{c}30000 \\
30000 \\
70000 \\
70000 \\
70000 \\
70000 \\
70000 \\
70000 \\
70000 \\
70000\end{array}$ & $\begin{array}{c}30000 \\
30000 \\
70000 \\
70000 \\
70000 \\
70000 \\
70000 \\
70000 \\
70000 \\
70000\end{array}$ & $\begin{array}{c}30000 \mathrm{~T} \\
30000 \\
70000 \\
70000 \\
70000 \\
70000 \\
70000 \\
70000 \\
70000 \\
70000\end{array}$ & $\begin{array}{c}30000 \\
30000 \\
70000 \\
70000 \\
70000 \\
70000 \\
70000 \\
70000 \\
70000 \\
70000\end{array}$ & $\begin{array}{c}30000 \\
30000 \\
70000 \\
-999 \\
70000 \\
70000 \\
70000 \\
70000 \\
70000 \\
70000\end{array}$ & $\begin{array}{c}30000 \\
30000 \\
70000 \\
-999 \\
70000 \\
70000 \\
70000 \\
-999 \\
70000 \\
70000\end{array}$ & $\begin{array}{l}70000 \\
70000 \\
70000 \\
70000\end{array}$ & $\begin{array}{l}70000 \\
70000 \\
70000 \\
70000\end{array}$ & $\begin{array}{l}70000 \\
70000 \\
70000 \\
70000\end{array}$ & $\begin{array}{l}30000 \\
70000 \\
70000 \\
70000 \\
70000\end{array}$ \\
\hline \multicolumn{19}{|c|}{ Last five records of the file: } \\
\hline $\begin{array}{l}44 \mathrm{LYHC} \\
30000 \\
44 \mathrm{LYHC} \\
30001 \\
44 \mathrm{LYHC} \\
30000 \mathrm{~T} \\
44 \mathrm{LYHC} \\
30000 \\
44 \mathrm{LYHC} \\
70000\end{array}$ & $\begin{array}{l}\text { SNWDTI19 } \\
30000 \\
\text { SNWDTI19 } \\
30000 T \\
\text { SNWDTI19 } \\
30000 \\
\text { SNWDTI19 } \\
30000 \\
\text { SNWDTI19 } \\
70000\end{array}$ & $\begin{array}{c}431231 \\
30000 \\
440131 \\
30000 \mathrm{~T} \\
440229 \\
30000 \\
440331 \\
30012 \\
440430 \\
70000\end{array}$ & $\begin{array}{c}30000 \\
30000 \\
30000 \mathrm{~T} \\
30000 \mathrm{~T} \\
30000 \\
30000 \\
30000 \\
30013 \\
70000 \\
70000\end{array}$ & $\begin{array}{c}30000 \\
30000 \\
30000 \mathrm{~T} \\
30000 \\
30000 \\
30000 \\
30000 \\
30000 \mathrm{~T} \\
70000 \\
70000\end{array}$ & $\begin{array}{c}30000 \\
30000 \\
30000 \mathrm{~T} \\
30000 \\
30000 \\
30000 \\
30000 \\
30000 \mathrm{~T} \\
70000 \\
70000\end{array}$ & $\begin{array}{c}30000 \\
30000 \\
30000 \\
30000 \\
30000 \\
30000 \\
30000 \\
30000 \\
70000 \\
70000\end{array}$ & $\begin{array}{c}30000 \\
30000 \\
30000 \\
30000 \\
30000 \\
30000 \\
30000 \\
30000 \\
70000 \\
70000\end{array}$ & $\begin{array}{c}30000 \\
30000 \mathrm{~T} \\
30000 \\
30000 \\
30000 \\
30000 \\
30000 \\
30000 \\
70000 \\
70000\end{array}$ & $\begin{array}{c}30000 \\
30000 \\
30000 \\
30000 \\
30000 \\
30000 \\
30000 \\
30000 \\
70000 \\
70000\end{array}$ & $\begin{array}{c}30000 \\
30000 \\
30004 \\
30000 \\
30000 \\
30000 \\
30000 \\
30000 \\
70000 \\
70000\end{array}$ & $\begin{array}{c}30000 \\
30000 \\
30001 \\
30000 \\
30010 \\
30000 \\
30000 \\
30000 \\
70000 \\
70000\end{array}$ & $\begin{array}{c}30000 \\
30017 \\
30000 \mathrm{~T} \\
30000 \\
30000 \mathrm{~T} \\
30000 \\
30000 \\
30000 \\
70000 \\
70000\end{array}$ & $\begin{array}{c}30000 \\
30012 \\
30000 \mathrm{~T} \\
30000 \\
30000 \mathrm{~T} \\
-999 \\
30000 \\
30000 \\
70000 \\
70000\end{array}$ & $\begin{array}{c}30000 \\
30001 \\
30000 \mathrm{~T} \\
30000 \\
30000 \mathrm{~T} \\
-999 \\
30000 \\
30000 \\
70000 \\
-999\end{array}$ & $\begin{array}{l}30000 \\
30000 \mathrm{~T}\end{array}$ & $\begin{array}{l}30000 \\
30000\end{array}$ & $\begin{array}{l}30000 \\
30000 \mathrm{~T} \\
30000 \mathrm{~T}\end{array}$ & $\begin{array}{l}30000 \\
30004 \\
30000 \% \\
30000\end{array}$ \\
\hline
\end{tabular}


Table 4 (continued)

\section{NEAST.DAT (FILE 13):}

First five records of the file:

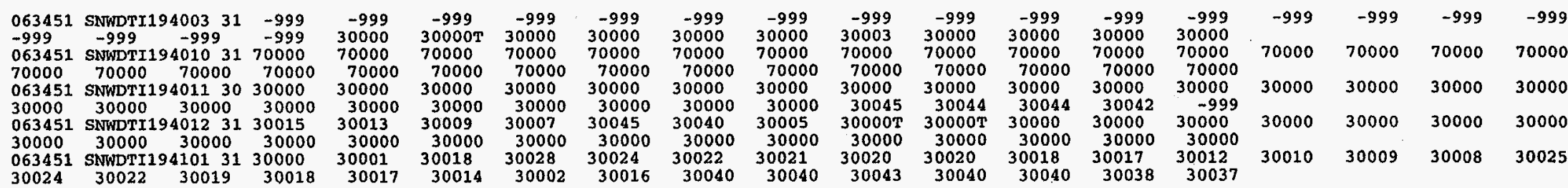

Last five records of the file:

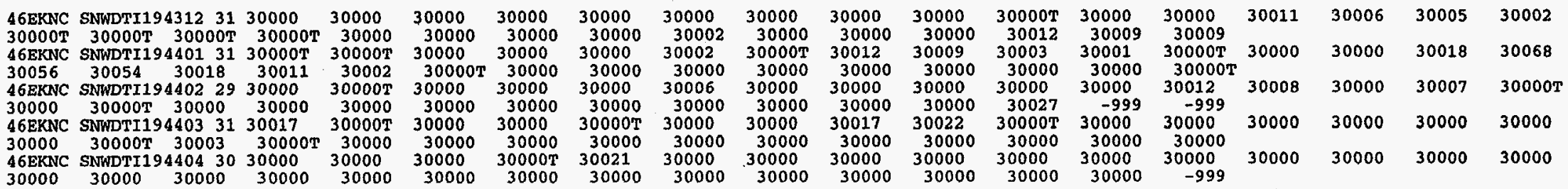


APPENDIX A

LIST OF STATE ABBREVIATIONS, NCDC STATE CODES, AND STATE NAMES 


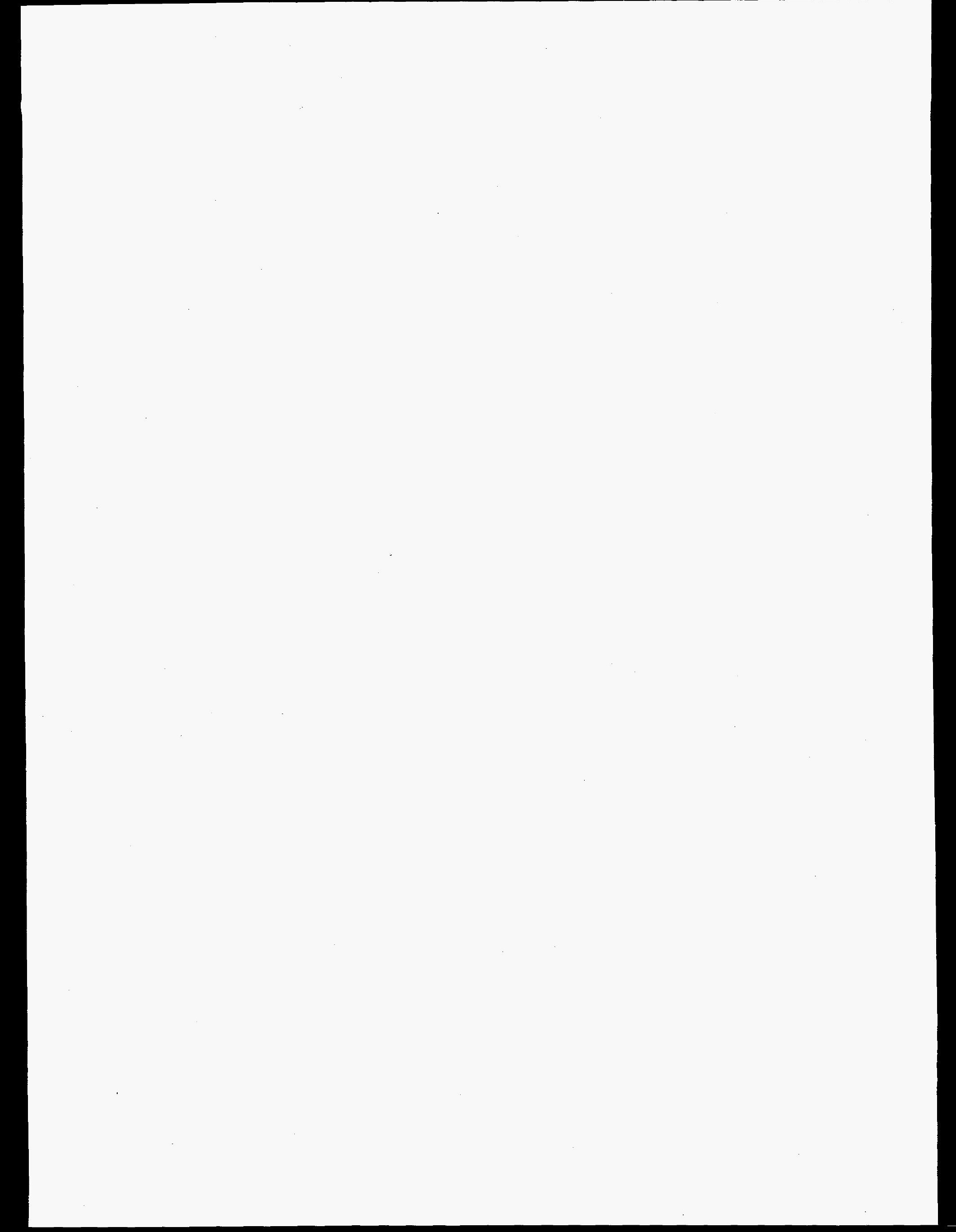




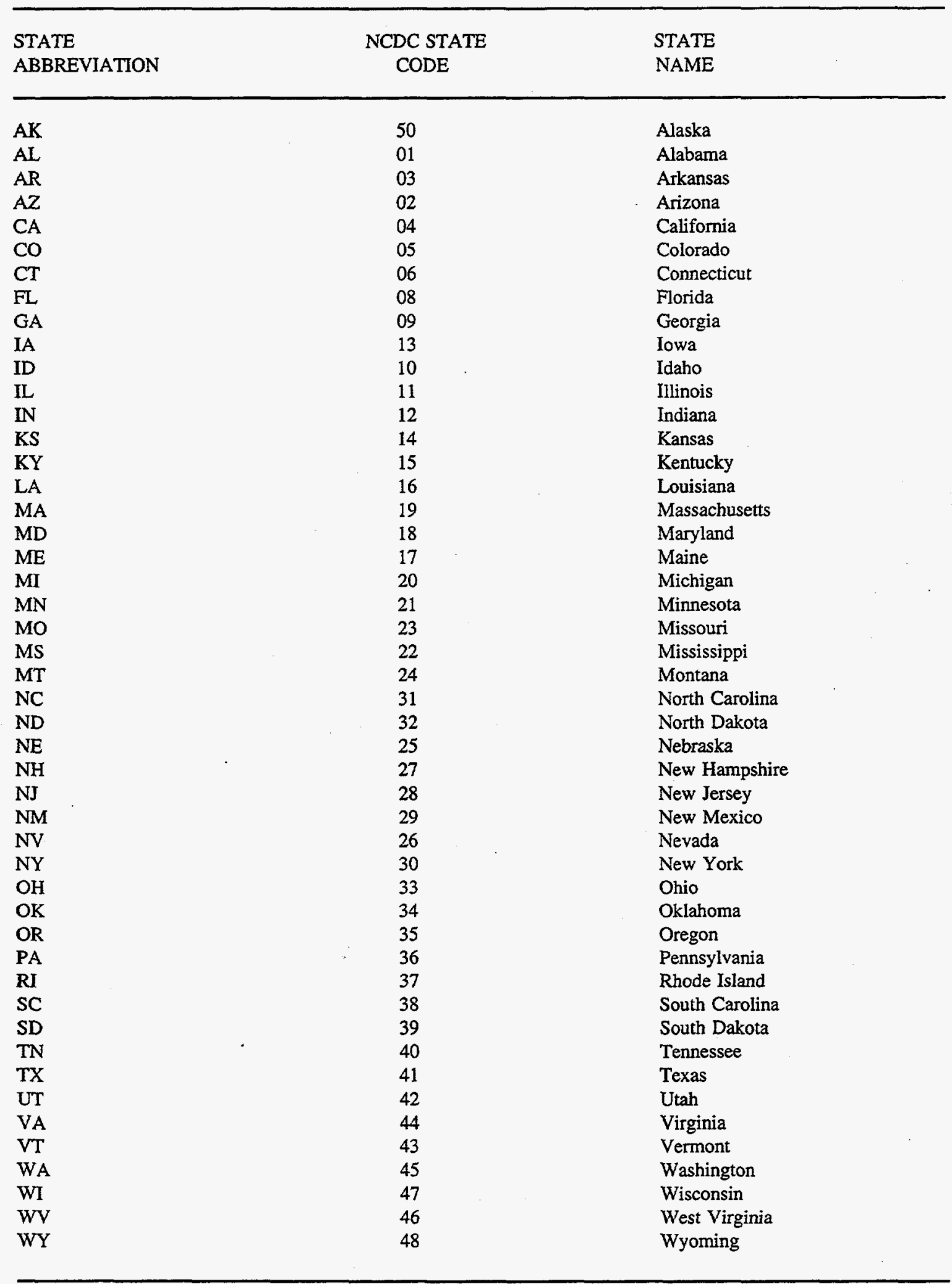




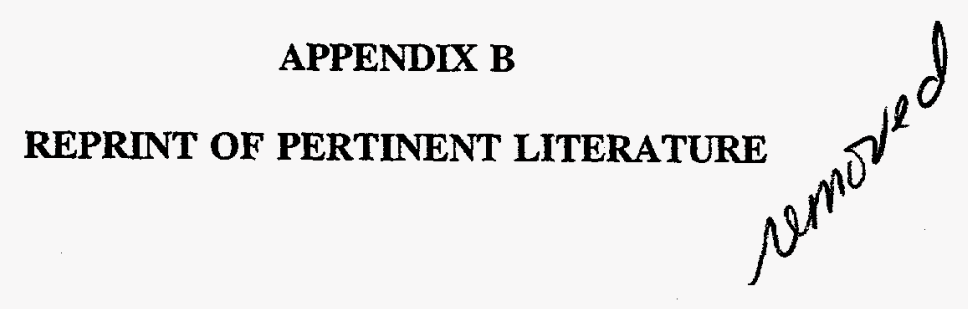




\section{INTERNAL DISTRIBUTION}

1. L. J. Allison

2. T. A. Boden

3. R. M. Cushman

4. S. V. Jennings

5. S. B. Jones

6. D. P. Kaiser

7. P. Kanciruk

8. A. W. King

9. T. E. Myrick
10. D. E. Shepherd

11. D. S. Shriner

12. L. D. Voorhees

13. Central Research Library

14-17. ESD Library

18-19. Laboratory Records Department ORNL- RC

20. Laboratory Records Department

21. Y-12 Technical Library

\section{EXTERNAL DISTRIBUTION}

22. Shelton S. Alexander, Pennsylvania State University, Department of Geosciences, 537 Deike Building, State College, PA 16802

23. Joe H. Allen, WDC-A for Solar Terrestrial Physics, National Geophysical Data Center, National Oceanic and Atmospheric Administration, E/GC2, 325 Broadway, Boulder, CO 80303

24. William E. Asher, University of Washington, Joint Institute for the Study of the Atmosphere and the Ocean, Box 354235, Seattle, WA 98195

25. David C. Bader, Pacific Northwest National Laboratory, 901 D Street, SW, Suite 900, Washington, DC 20024

26. Roger G. Barry, University of Colorado, WDC-A Glaciology, CIRES, Campus Box 449, Boulder, CO 80309

27. Marion F. Baumgardner, Purdue University, Department of Agronomy, 1220 Potter Drive, Lafayette, IN 47907

28. Robert Bidigare, University of Hawaii, Department of Oceanography, 1000 Pope Road, Honolulu, HI 96822

29. C. Boelcke, P.S.S., UNEP, P.O. Box 30552, Nairobi, Kenya

30. D. P. Bowman, National Oceanic and Atmospheric Administration, National Climatic Data Center, 151 Patton Avenue, Suite 120, Asheville, NC 28801

31. Francis P. Bretherton, University of Wisconsin, Space Science and Engineering Center, 1225 West Dayton Street, Madison, WI 53706 
32. Peter G. Brewer, Monterey Bay Aquarium Research Institute, P.O. Box 628, 7700 Sandholt Rd., Moss Landing, CA 95039

33. Michelle Broido, Department of Energy, Office of Health and Environmental Research, Environmental Sciences Division, ER-74, 19901 Germantown Road, Germantown, MD 20874

34. D. A. Bruns, Wilkes University, Department of Earth and Environmental Sciences, Wilkes-Barre, PA 18766

35. Robert H. Byrne, University of South Florida, Department of Marine Science, 140 Seventh Avenue, Saint Petersburg, FL 33701

36. D. Changnon, Northern Illinois University, Department of Geography, DeKalb, IL 60115-2854

37. Patrick A. Crowley, Department of Energy, Office of Health and Environmental Research, Environmental Sciences Division, ER-74, 19901 Germantown Road, Germantown, MD 20874

38. H. Croze, P.S.S., UNEP, P.O. Box 30552, Nairobi, Kenya

39. E. G. Cumesty, ORNL Site Manager, Department of Energy, Oak Ridge National Laboratory, P.O. Box 2008, Oak Ridge, TN 37831-6269

40. Roger C. Dahlman, Department of Energy, Office of Health and Environmental Research, Environmental Sciences Division, ER-74, 19901 Germantown Road, Germantown, MD 20874

41. Giovanni Daneri, CEA Universidad del Mar, Department de Oceanografia y Biologia Pesquera, Amunategui 1838, Vina Del Mar, Chile

42. Database Section, National Institute for Environmental Studies, Center for Global Environmental Research, 16-2 Onogawa, Tsukuba, Ibaraki 305, Japan

43. Kenneth D. Davidson, National Climatic Data Center, 151 Patton Avenue, Suite 120, Asheville, NC 28801

44. William C. Draeger, WDC-A for Remotely Sensed Land Data, U.S. Geological Survey, EROS Data Center, Sioux Falls, SD 57198

45. Hugh W. Ducklow, College of William and Mary, Virginia Institute of Marine Sciences, P.O. Box 1346, Gloucester Point, VA 23062 
46-50. D. R. Easterling, National Oceanic and Atmospheric Administration, National Climatic Data Center, 151 Patton Avenue, Suite 120, Asheville, NC 28801

51. Jerry W. Elwood, Department of Energy, Office of Energy Research, ER-74, 19901 Germantown Road, Germantown, MD 20874

52. Lawrence M. Enomoto, National Oceanic and Atmospheric Administration, NESDIS, Federal Building 4, Room 0110, Washington, DC 20233

53. Gerd Esser, Justus-Liebig-University, Institute for Plant Ecology, Heinrich-BuffRing 38, D-35392, Giessen, Germany

54. Jay S. Fein, National Science Foundation, Atmospheric Science Division, 4201 Wilson Boulevard, Room 775, Arlington, VA 22230

55. Wanda Ferrell, Department of Energy, Office of Health and Environmental Research, Environmental Sciences Division, ER-74, 19901 Germantown Road, Germantown, MD 20874

56. B. F. Findlay, Environment Canada, Atmospheric Environment Service, Climate Research Branch, Downsview, Ontario Canada M3H5T4

57. Richard H. Gammon, University of Washington, Chemistry Department, Box 351700 , Seattle, WA 98195

58. Wilford Gardner, Texas A \& M University, Department of Oceanography, College Station, TX 77843

59. Jean-Pierre Gattuso, Observatoire Oceanologique Europeen, Avenue Saint-Martin, MC-98000, Monaco

60. P. Ya. Groisman, National Oceanic and Atmospheric Administration, National Climatic Data Center, 151 Patton Avenue, Suite 120, Asheville, NC 28801

61. D. W. Gullett, Environment Canada, Atmospheric Environment Service, Climate Research Branch, Downsview, Ontario Canada M3H5T4

62. Kenneth D. Hadeen, National Climatic Data Center, 151 Patton Avenue, Suite 120, Asheville, NC 28801

63. David O. Hall, University of London, Division of Biosphere Sciences, King's College London, Campden Hill Road, London W8 7AH, United Kingdom

64. Mark Hein, Freshwater Biological Laboratory, Helsingoersgade 51, DK-3400 Hilleroed, Denmark 
65. A. Hittelman, WDC-A for Solid Earth Geophysics, National Oceanic and Atmospheric Administration Code E/GC1, 325 Broadway, Boulder, CO 80303

66. H. Hodgson, British Library, Boston Spa, DSC, Special Acquisitions, Wetherby, West Yorkshire, LS23 7BQ, United Kingdom

67. Huasheng Hong, Xiamen University, Environmental Science Research Center, Post Code 361005, Mail Box 1085, Xiamen, Fujian, Peoples Republic China

68. Carroll A. Hood, GCRIO, 2250 Pierce Road, Bay City, MI 48710

69. John C. Houghton, Department of Energy, Office of Health and Environmental Research, Environmental Sciences Division, ER-74, 19901 Germantown Road, Germantown, MD 20874

70. Malcolm K. Hughes, University of Arizona, Laboratory of Tree-Ring Research, Building 58, $1105 \mathrm{~W}$. Stadium, Tucson, AZ 85721

71. P. Y. Hughes, National Ocean and Atmospheric Administration, National Climatic Data Center, 151 Patton Avenue, Suite 120, Asheville, NC 28801

72. M. Hulme, Climatic Research Unit, School of Environmental Sciences, University of East Anglia, Norwich NR47TJ, United Kingdom

73. P. Jamason, National Oceanic and Atmospheric Administration, National Climatic Data Center, 151 Patton Avenue, Suite 120, Asheville, NC 28801

74. R. Jenne, National Center for Atmospheric Research, P.O. Box 3000, 1850 Table Mesa Drive, Boulder, CO 80307-3000

75. P. D. Jones, Climatic Research Unit, School of Environmental Sciences, University of East Anglia, Norwich NR47TJ, United Kingdom

76. Thomas R. Karl, National Climatic Data Center, 151 Patton Avenue, Federal Building, Room 516E, Asheville, NC 28801

77. Charles D. Keeling, Scripps Institute of Oceanography, University of California, San Diego, Mail Code 0220, 9500 Gilman Drive, 2314 Ritter Hall, La Jolla, CA 92093

78. Stephan Kempe, Schnittspahnstr. 9, D-64287 Darmstadt, Germany

79. R. M. Key, Princeton University, Geology Department, Princeton, NJ 08544

80. K.-R. Kim, Seoul National University, Department of Oceanology, Seoul 151-7442, Korea 
81. Takashi Kimoto, Research Institute of Oceano-Chemistry, Osaka Office, 3-1 Fumahashi-cho, Tennoji-ku, Osaka 543, Japan

82. Bert Klein, University Laval, GIROQ, Pav. Vachon, Quebec, PQ, G1K 7P4, Canada

83. John C. Klink, Miami University, Department of Geography, 217 Shideler Hall, Oxford, $\mathrm{OH} 45056$

84. R. W. Knight, National Oceanic and Atmospheric Administration, National Climatic Data Center, 151 Patton Avenue, Suite 120, Asheville, NC 28801

85. Werner A. Kurz, Environmental and Social Systems Analysts Limited, 3rd Floor, 1765 W 8th Avenue, Vancouver, B.C. V6J 5C6, Canada

86. Donald T. Lauer, EROS Data Center, U.S. Geological Survey, Sioux Falls, SD 57198

87. J. D. Laver, Climate Prediction Center, National Oceanic and Atmospheric Administration, National Weather Service, 5200 Auth Road, Room 800, Camp Springs, MD 20746

88. Sydney Levitus, National Oceanographic Data Center, National Oceanic and Atmospheric Administration, E/OC5, 1315 East West Highway, Room 4362, Silver Spring, MD 20910

89. Jonathan J. Lloyd, Australian National University, Research School of Biological Sciences, GPO Box 475, Canberra, ACT 2601, Australia

90. Peter Lunn, Department of Energy, Office of Health and Environmental Research, Environmental Sciences Division, ER-74, 19901 Germantown Road, Germantown, MD 20874

91. Thomas H. Mace, U.S. Environmental Protection Agency, National Data Processing Division, MD-34, 79 TW Alexander Drive, Building 4201, Durham, NC 27711

92. E. H. Mason, National Oceanic and Atmospheric Administration, National Climatic Data Center, 151 Patton Avenue, Suite 120, Asheville, NC 28801

93. Elaine Matthews, National Aeronautics and Space Administration, Goddard Institute for Space Studies, 2880 Broadway, New York, NY 10025

94. James J. McCarthy, Harvard University, Museum of Comparative Zoology, 26 Oxford Street, Cambridge, MA 02138 
95. Michael C. McCracken, Director, Office of the U.S. Global Change Research Program, Code YS-1, 300 E. Street, SW, Washington, DC 20546

96. Frank J. Millero, University of Miami, RSMAS, 4600 Rickenbacker Causeway, Miami, FL 33149

97. R. E. Munn, University of Toronto, Institute for Environmental Studies, Haultain Building 170 College Street, Toronto, Ontario M5S 1A4, Canada

98. Shohei Murayama, National Institute for Resources and Environment, Environmental Assessment Department, 16-3 Onogawa, Tsukuba, Ibaraki 305, Japan

99. Shuzo Nishioka, National Institute for Environmental Studies, Global Environment Research Division, 16-2 Onogawa, Tsukuba, Ibaraki 305, Japan

100. Jao Ryoung Oh, Korea Ocean Research and Development Institute, Chemical Oceanography Division, An San P.O. Box 29, Seoul 4325-600, Korea

101. C. Oudot, Centre ORSTOM de Cayenne, B.P. 165-97323, Cayene Cedex, Guyana

102. Bobbi Parra, Department of Energy, Office of Health and Environmental Research, Environmental Sciences Division, ER-74, 19901 Germantown Road, Germantown, MD 20874

103. William Parton, Colorado State University, Natural Resource Ecology Laboratory, Fort Collins, $\mathrm{CO} 80523$

104. Ari Patrinos, Department of Energy, Office of Health and Environmental Research, Environmental Sciences Division, ER-74, 19901 Germantown Road, Germantown, MD 20874

105. Tsung-Hung Peng, National Oceanographic and Atmospheric Administration, Atlantic Oceanographic Marine Laboratory, Ocean Chemistry Division, 4301 Rickenbacker Causeway, Miami, FL 33149

106. Fiz F. Perez, Instituto Investigacions Marinas, Eduardo Cabello 6, 36206 Vigo, Spain

107. B. Preselin, University of California, Department of Biological Sciences, Santa Barbara, CA 93106

108. Paul D. Quay, University of Washington, School of Oceanography, Box 357940 , Seattle, WA 98195

109. Roberta Y. Rand, USDA, Global Change Data and Information Management, 10301 Baltimore Boulevard, Beltsville, MD 20705 
110. S. Ichtiaque Rasool, 52, Boulevard de Sebastopol, 5th Floor, 75003 Paris, France

111. Joachim Ribbe, University of Washington, Joint Institute for the Study of the Atmosphere and Oceans, Box \# 35425, Seattle, WA 98195

112. Michael R. Riches, Department of Energy, Office of Health and Environmental Research, Environmental Sciences Division, ER-74, 19901 Germantown Road, Germantown, MD 20874

113. Aida F. Rios, Institute de Investigaciones Marinas, Consejo Superior de Invest. Cientificas, c/Eduardo Cabello, 6, 36208 Vigo, Spain

114. D. Robinson, Lucy Stone Hall, Department of Geography, Kilmer Campus, New Brunswick, NJ 08903

115. A. Robock, University of Maryland, Department of Meteorology, College Park, MD 20742

116. Jorge L. Sarmiento, Princeton University, Atmospheric and Oceanic Sciences Program, P.O. Box CN710, Sayre Hall, Princeton, NJ 08544

117. C. L. Schelske, University of Florida, Department of Fisheries and Aquaculture, 7922 W. 71 st Street, Gainesville, FL 32606

118. Soroosh Sorooshian, University of Arizona, Department of Hydrology and Water Resources, Tucson, AZ 85721

119-218. Timothy W. Stamm, CDIAC, The University of Tennessee, EERC, 10521 Research Drive, Suite 100, Knoxville, TN 37923

219. Michel H. C. Stoll, Netherlands Institute for Sea Research, Department MCG, P. O. Box 59, $1790 \mathrm{Ab}$ den Burg-Texel, Netherlands

220. Eric T. Sundquist, U.S. Geological Survey, Quissett Campus, Branch of Atlantic Marine Geology, Woods Hole, MA 02543

221. Taro Takahashi, Columbia University, Lamont-Doherty Earth Observatory, Climate/ Environment/Ocean Division, Route 9W, Palisades, NY 10964

222. Lynne D. Talley, Scripps Institute of Oceanography, UCSD 0230, 9500 Gilman Drive, Solana Beach, CA 92075

223. John A. Taylor, Australian National University, CRES, GPO Box 4, Canberra, ACT 0200, Australia 
224. John R. G. Townshend, University of Maryland, Department of Geography, 1113 Lefrak Hall, College Park, MD 20742

225. K. Vinnikov, University of Maryland, Department of Meteorology, College Park, MD 20742

226. Douglas W. R. Wallace, Brookhaven National Laboratory, Oceanographic and Atmospheric Sciences Division, P.O. Box 5000, Upton, NY 11973

227. Carol Watts, National Oceanic and Atmospheric Administration, Central Library, 1315 East-West Highway, 2nd Floor, SSMC 3, Silver Spring, MD 20910

228. Thompson Webb III, Brown University, Department of Geological Sciences, 324 Brook Street, Providence, RI 02912

229. Ferris Webster, University of Delaware, College of Marine Studies, Lewes, DE 19958

230. Amos Winter, University of Puerto Rico, Department of Marine Sciences, Puerto Rico State Climate Office, P.O. Box 5000, Mayaguez, PR 00681

231. Chi Shing Wong, Government of Canada, Institute of Ocean Sciences, P.O. Box 6000,9860 West Saanich Road, Sidney, BC V8L 4B2, Canada

232. Yoshifumi Yosuoka, National Institute for Environmental Studies, Center Global Environment Research, 16-2 Onogawa, Tsukuba, Ibaraki 305, Japan

233. Energy Library (HR-832.1/GTN), Department of Energy, Office of Administration and Management, G-034, Washington, DC 20585

234. Energy Library (HR-832.2/WAS), Department of Energy, Office of Administration and Management, GA-138 Forrestal Building, Washington, DC 20585

235-236. Office of Scientific and Technical Information, P. O. Box 62, Oak Ridge, TN 37831

237. Office of Assistant Manager for Energy Research and Development, Department of Energy, Oak Ridge Operations, P. O. Box 2001, Oak Ridge, TN 37831-8600 